\title{
The influence of strain rate and presence of dispersed second phases on the deformation behaviour of polycrystalline $\mathrm{D}_{2} \mathrm{O}$ ice
}

\author{
CHRISTOPHER J.L. WILSON, ${ }^{1}$ (ㅇ NICHOLAS J. R. HUNTER, ${ }^{1}$ VLADIMIR LUZIN, ${ }^{2}$ \\ MARK PETERNELL, ${ }^{3}$ SANDRA PIAZOLO ${ }^{4}$ \\ ${ }^{1}$ School of Earth, Atmosphere and Environment, Monash University, Clayton, Victoria 3800, Australia \\ ${ }^{2}$ Australian Centre for Neutron Scattering, Australian Nuclear Science and Technology Organisation, Lucas Heights, \\ NSW 2234, Australia \\ ${ }^{3}$ Institute of Geosciences, University of Mainz, 55128 Mainz, Germany \\ ${ }^{4}$ School of Earth and Environment, University of Leeds, Leeds, UK \\ Correspondence: Christopher J.L. Wilson <Chris.Wilson@monash.edu>
}

\begin{abstract}
This contribution discusses results obtained from 3-D neutron diffraction and 2-D fabric analyser in situ deformation experiments on laboratory-prepared polycrystalline deuterated ice and ice containing a second phase. The two-phase samples used in the experiments are composed of an ice matrix with (1) air bubbles, (2) rigid, rhombohedral-shaped calcite and (3) rheologically soft, platy graphite. Samples were tested at $10^{\circ} \mathrm{C}$ below the melting point of deuterated ice at ambient pressures, and two strain rates of $1 \times 10^{-5} \mathrm{~s}^{-1}$ (fast) and $2.5 \times 10^{-6} \mathrm{~s}^{-1}$ (medium). Nature and distribution of the second phase controlled the rheological behaviour of the ice by pinning grain boundary migration. Peak stresses increased with the presence of second-phase particles and during fast strain rate cycles. Ice-only samples exhibit well-developed crystallographic preferred orientations (CPOs) and dynamically recrystallized microstructures, typifying deformation via dislocation creep, where the CPO intensity is influenced in part by the strain rate. CPOs are accompanied by a concentration of [c]-axes in cones about the compression axis, coinciding with increasing activity of prismatic- $<\mathbf{a}>$ slip activity. Ice with second phases, deformed in a relatively slower strain rate regime, exhibit greater grain boundary migration and stronger CPO intensities than samples deformed at higher strain rates or strain rate cycles.
\end{abstract}

KEYWORDS: ice crystal studies, ice rheology, structural glaciology

\section{INTRODUCTION}

The correct interpretation of microstructure and crystallographic preferred orientations (CPOs) in terms of deformation mechanisms and flow properties is critical in understanding the bulk behaviour of polycrystalline ice in glaciers and ice sheets. This relies on the patterns and strength of the CPO together with grain size and distribution of impurities (Hooke and others, 1972; Moore, 2014). In pure ice, a commonly accepted view is that it behaves like a non-Newtonian fluid, dominantly controlled by 'grain-size insensitive' creep mechanisms, such as dislocation glide and climb (de Bresser and others, 1998). However, this simplification discounts the complex stress and strain partitioning processes that may occur within ice containing included debris and micro-particle content (Alley and others, 1986; Fisher and Koerner, 1986; Li and others, 1998; Eichler and others, 2017), or dust and soluble-ion concentrations (Thorsteinsson and others, 1999; Durand and others, 2006). The microstructural complexities associated with interpreting ice with solid inclusions is due primarily to the coupled roles of extrinsic (stress and temperature) and intrinsic (physical components within the ice mass), which lead to heterogeneous strain accommodation across ice masses (Thorsteinsson and others, 1997). In impure ice containing rheologically differing phases, extrinsic and intrinsic factors (e.g. proportion of and stress portioning into second phase, phase connectivity, ductility) are coupled (Alley and others, 1986). This means that deformation will be recorded differently between pure and impure ice forms, which Shoji and Langway (1988) argue is primarily controlled by the orientation strength of [c]-axes. In nature, this may be further complicated where variable strain rates are superimposed on a pre-existing grain structure by faster flowing portions of a glacier or ice sheet.

In most of today's large-scale ice-sheet numerical models, ice is treated as a single constituent or homogeneous material where the flow laws are employed throughout the entire ice sheet (e.g. Alley, 1992; Gagliardini and others, 2013; Martin and others, 2014). However, this simplification is not confirmed by observations, as borehole closures and tilt measurements in many ice sheets can be correlated with second-phase content (Fisher and Koerner, 1986), and also to changes in CPOs (Shoji and Langway, 1984; Tison and others, 1994). CPOs have also been included in some glacier or ice-sheet numerical models relating strain and fabric evolution (e.g. Castelnau and others, 1998; GilletChaulet and others, 2006; Pettit and others, 2007; Martin and others, 2009); although plausible these have been incompletely verified by experimental observations. It is known that ice sheets are complex as they have high strain rates at the lateral and basal margins, which may contain entrained debris, formed under stress conditions much different than the fairly well-studied ones near ice divides (Thorsteinsson and others, 1997; Tison and others, 1994). Therefore, there are significant challenges remaining as there are few experimental studies that offer direct information about rheology, microstructure and $\mathrm{CPO}$ changes 
Table 1. Examples of some previous laboratory observations on the role of particulate second phases on ice creep behaviour

\begin{tabular}{|c|c|c|}
\hline $\begin{array}{l}\text { Role of particulate second phase on creep } \\
\text { behaviour in ice }\end{array}$ & Nature of second phase & Reference \\
\hline Leads to mechanical hardening & $\begin{array}{l}\text { Sand; } \mathrm{SiO}_{2} \text { particles } \mathrm{SiC} \text { and } \\
\mathrm{CaCO}_{3} \text { Fluorite }\end{array}$ & $\begin{array}{l}\text { Sayles (1968); Nayar and others (1971); Hooke and others (1972); } \\
\text { Durham and others (1992); Middleton and others (2017) }\end{array}$ \\
\hline Leads to mechanical weakening & $\begin{array}{l}\text { Silt-sized soil particles Low } \\
\text { sand concentrations }\end{array}$ & Hooke and others (1972); Song and others $(2005,2008)$ \\
\hline No influence on mechanical behaviour & $\begin{array}{l}<15 \% \text { quartz sand Small vol. } \\
\% \text { of silica beads }\end{array}$ & Jacka and others (2003); Yasui and Arakawa (2008) \\
\hline $\begin{array}{l}\text { Creep behaviour depends on nature, percent- } \\
\text { age of second phase and temperature range }\end{array}$ & Calcite, graphite, silica beads & $\begin{array}{l}\text { Hooke and others (1972); Baker and Gerberich (1979); Yasui and } \\
\text { Arakawa (2008); Cyprych and others (2016); Peternell and Wilson } \\
\text { (2016) }\end{array}$ \\
\hline
\end{tabular}

The variations in creep rates in many of these earlier studies are the result of particulate grain size or compositional differences between samples and variable inclusion concentrations.

across a strain gradient and particularly if the ice contains a dispersed second phase. There are also conflicting views on the role played by particulate second phases on the creep behaviour of ice (Table 1). This leads to major uncertainties as to the validity of microstructural interpretations of particulate distributions within ice cores.

Cyclic or non-steady state deformation involving changing differential stresses or oscillations in the strain rate gradients are also likely to be common in nature (Kamb and Engelhardt, 1987; Joughin and others, 2010), even though most ice experimental studies have involved constant load or strain rate experiments (e.g. Durham and others, 1988; Dahl-Jensen and others, 1997; Jacka and Li, 2000; Piazolo and others, 2013). To date there are few experimental comparisons (e.g. Peternell and Wilson, 2016) involving fluctuations in strain rate, in which the instantaneous strain rate may exceed the average strain rate. These experiments give some insight into more complicated histories (Bindschadler and others, 2013). Similarly the effect of strain rate and initial grain size in pure ice may influence primary creep rates, whereas subsequent tertiary creep appears to be unaffected by the initial grain size (Duval and Le Gac, 1980). However, there is still a lack of data on grain size effects in ice containing dispersed phases, which can be explained by arguments related to the operation of dislocation mechanisms rather than from elastic anisotropy (Schulson and Duval, 2009).

To investigate the effect of coupling a second phase to variations in strain rate, we will compare a series of 3-D and 2-D in situ experiments (Fig. 1) involving pure ice and ice containing rheologically soft and rigid particles. In the 2-D experiments, a comparison will also be made between ice flowing at a constant strain rate to short-term cycling events and their feedback into grain size and CPO development. In all experiments, we have synchronously and continuously recorded strain distributions, $\mathrm{CPO}$ and grain size evolution at incremental strains up to $20 \%$ shortening.

\section{METHODS}

All experiments (Table 2) have used a matrix of deuterated ice $\left(\mathrm{D}_{2} \mathrm{O}\right)$, as this allows quantitative in situ neutron diffraction and grain size analysis during deformation. $\mathrm{D}_{2} \mathrm{O}$ has the same crystal structure, similar mechanical properties, and deformation behaviour as $\mathrm{H}_{2} \mathrm{O}$ ice (Petrenko and Whitworth, 1999; McDaniel and others, 2006; Wilson and others, 2014; Middleton and others, 2017). Undeformed samples of deuterated ice were prepared by crushing and mixing 180 $250 \mu \mathrm{m}$ sieved ice particles with liquid $\mathrm{D}_{2} \mathrm{O}$ at -10 to $-5^{\circ} \mathrm{C}$ within moulds. Air bubbles are mechanically expelled by stirring the mixture and excess water removed by lightly compressing the sample prior to freezing and annealing for a month at $-10^{\circ} \mathrm{C}$. The microstructure in the pure ice is dominated by polygonal grains with straight grain boundaries, a low porosity, mean grain size $\sim 400-300 \mu \mathrm{m}$ (Fig. 2a). Fabric analysis revealed $[c]$-axis fabrics of the undeformed ice (e.g. Figs 2a,

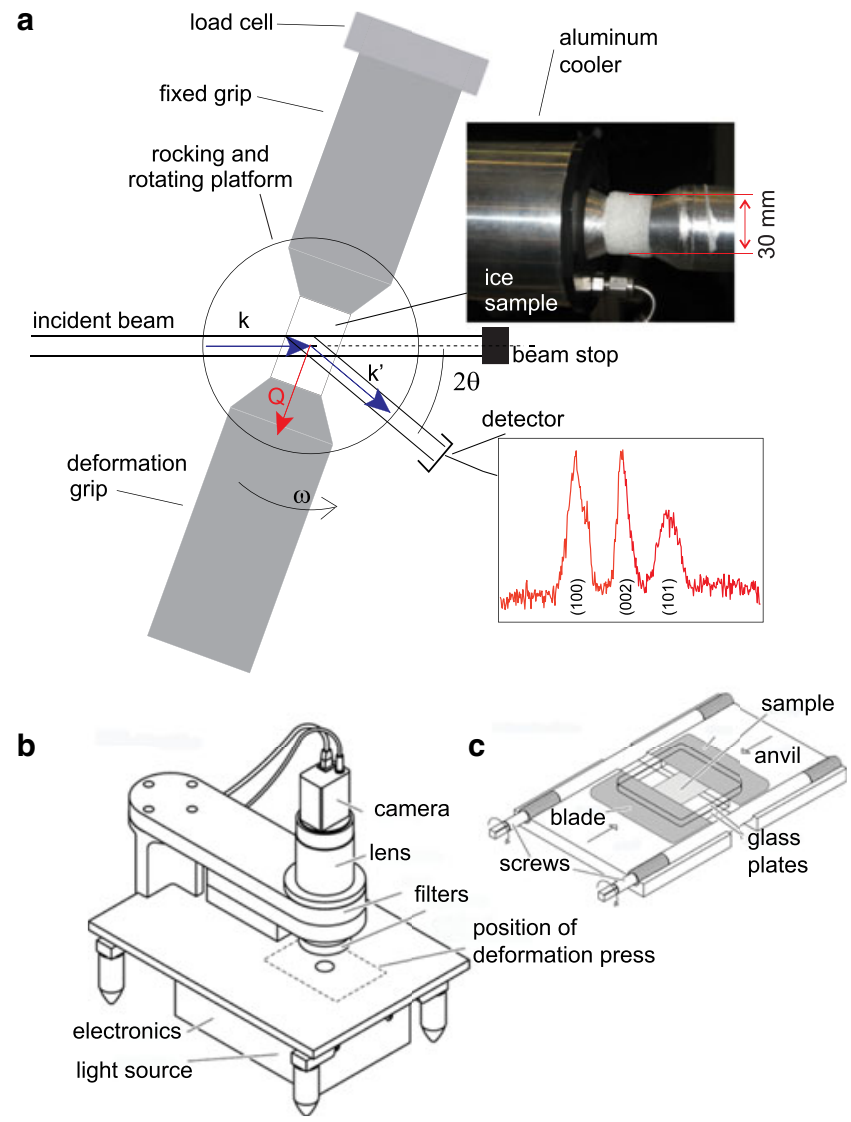

Fig. 1. Schematic illustrations showing the deformation set-up in the 3-D and 2-D experiments. (a) The Kowari instrument configuration for 3-D neutron diffraction and CPO analysis. Insets show deformed ice sample, from an initial sample that had a diameter $\sim 2.5 \mathrm{~cm}$, length $\sim 3.2-4.0 \mathrm{~cm}$ and ice diffraction pattern. (b) Sketch of G50 fabric analyser. (c) Sketch of 2-D deformation press with outer covers and upper halves of anvils removed for clarity. 
Table 2. Summary of experiments on deuterated $\left(\mathrm{D}_{2} \mathrm{O}\right)$ ice showing sample characteristics and deformation conditions

\begin{tabular}{|c|c|c|c|c|c|c|c|c|}
\hline \multirow[t]{2}{*}{ Sample } & \multirow[t]{2}{*}{$\begin{array}{l}\text { Second phase } \\
\text { type and vol. \% }\end{array}$} & \multirow{2}{*}{$\begin{array}{l}\text { Second phase } \\
\text { grain size } \\
\qquad \mu \mathrm{m}\end{array}$} & \multirow{2}{*}{$\begin{array}{l}\text { Time interval partial } \\
\text { pole figure } \\
\text { min }\end{array}$} & \multicolumn{2}{|c|}{$\begin{array}{l}\text { Mean neutron diffraction } \\
\text { ice grain size } \\
\mu \mathrm{m}\end{array}$} & \multirow{2}{*}{$\begin{array}{c}\text { Optical ice } \\
\text { grain size } \\
\mu \mathrm{m} \\
\text { Final }\end{array}$} & \multirow{2}{*}{$\begin{array}{c}\text { Strain rate } \\
\mathrm{s}^{-1}\end{array}$} & \multirow{2}{*}{$\begin{array}{c}\text { Strain } \\
\%\end{array}$} \\
\hline & & & & Starting & Final & & & \\
\hline D5-2 & Pure ice - & - & $\mathrm{N} / \mathrm{A}$ & 200-1000* & & & Starting material & 0 \\
\hline MD04 & Pure ice - & - & 20 & $447 \pm 61$ & $173 \pm 16$ & $271 \pm 45^{\dagger}$ & $1.0 \times 10^{-05}$ & 20 \\
\hline MD10 & Pure ice - & - & 35 & $474 \pm 46$ & $310 \pm 43$ & $246 \pm 9^{\dagger}$ & $2.5 \times 10^{-06}$ & 10 \\
\hline D1-7 & Pure ice - & - & 35 & $509 \pm 70$ & $272 \pm 30$ & $200-300$ & $2.5 \times 10^{-06}$ & 20 \\
\hline 13_31 & Bubble ice - & - & N/A & $100-1000^{*}$ & & & Starting material & 0 \\
\hline $13 \_32$ & Bubble ice - & - & 20 & Not & Analysed & $200-400$ & $1.0 \times 10^{-05}$ & 10 \\
\hline MD̄8 & Bubble ice - & _- & 35 & Not & Analysed & $300-500$ & $2.5 \times 10^{-06}$ & 10 \\
\hline MD11 & Bubble ice - & - & 20 & Not & Analysed & $372 \pm 14^{\dagger}$ & $1.0 \times 10^{-05}$ & 36 \\
\hline CC_03 & Coarse calcite 20 & $150-355$ & $\mathrm{~N} / \mathrm{A}$ & $200-600^{*}$ & No analysis & & Starting material & 0 \\
\hline CC_06 & Coarse calcite 20 & $150-355$ & 20 & $270 \pm 26$ & $173 \pm 16$ & $100-250$ & $1.0 \times 10^{-05}$ & 20 \\
\hline CC_05 & Coarse calcite 20 & 150-355 & 35 & $273 \pm 36$ & $170 \pm 13$ & $201 \pm 7^{\dagger}$ & $2.5 \times 10^{-06}$ & 20 \\
\hline FC_03 & Fine calcite 20 & $<150$ & $N / A$ & $100-600 *$ & No analysis & & Starting material & 0 \\
\hline FC_07 & Fine calcite 20 & $<150$ & 20 & $217 \pm 22$ & $147 \pm 14$ & $50-200$ & $1.0 \times 10^{-05}$ & 20 \\
\hline FFC_04 & Fine calcite 40 & $<150$ & 35 & $244 \pm 25$ & $137 \pm 19$ & $50-500$ & $2.5 \times 10^{-05}$ & 20 \\
\hline D5_1 & Coarse calcite 40 & $150-355$ & 35 & $483 \pm 48$ & $150 \pm 20$ & $N / A^{\ddagger}$ & $2.5 \times 10^{-05}$ & 40 \\
\hline G_02 & Graphite 20 & $150-355$ & 20 & $211 \pm 20$ & $148 \pm 23$ & $30-300$ & $1.0 \times 10^{-05}$ & 20 \\
\hline G_04 & Graphite 20 & $150-355$ & 35 & $231 \pm 21$ & $166 \pm 14$ & $100-500$ & $2.5 \times 10^{-06}$ & 6 \\
\hline G_06 & Graphite 20 & $150-355$ & 35 & $215 \pm 21$ & $146 \pm 17$ & $237 \pm 6^{\dagger}$ & $2.5 \times 10^{-06}$ & 20 \\
\hline GG_03 & $\begin{array}{l}\text { Graphite } 40 \\
\text { 2-D experiments }\end{array}$ & $\begin{array}{l}<150 \\
\text { Using FA }\end{array}$ & 35 & Not & Analysed & $82-135^{\dagger}$ & $2.5 \times 10^{-06}$ & 20 \\
\hline 18_4 & Pure ice 20 & Constant & $N / A$ & Not & Analysed & $320-340$ & $2.5 \times 10^{-06}$ & 20 \\
\hline $13 \_25$ & Pure ice 20 & Long cycle & $\mathrm{N} / \mathrm{A}$ & Not & Analysed & $320-380$ & $10.2-1.45 \times 10^{-06}$ & 20 \\
\hline D1_8 & Pure ice 20 & Short cycle & $\mathrm{N} / \mathrm{A}$ & Not & Analysed & $50-250$ & $2.8-1.9 \times 10^{-06}$ & 20 \\
\hline D-5_4 & Ice-calcite 40 & Short cycle & $\mathrm{N} / \mathrm{A}$ & Not & Analysed & $50-260$ & $2.8-1.9 \times 10^{-06}$ & 20 \\
\hline
\end{tabular}

All experiments were conducted at $-7 \mathrm{C}$, which is a direct analogue for deforming $\mathrm{H}_{2} \mathrm{O}$ ice at $-10 \mathrm{C}$ (experiment $\mathrm{H} 1 \_1$; illustrated in Fig. 7). The neutron diffraction starting grain size was calculated at $0.3 \%$ strain and changes in strain and grain sizes (Fig. 12) were obtained at specific time (minutes) intervals during an experiment. The range of optically measured grain sizes were measured from fabric analyser images.

* Grain size measured from optical image.

$\dagger$ Grain size measured from digitized image.

* Sample annealed after deformation.

3a, 4a) was close to random with minimal intracrystalline distortion within the grains.

For the second-phase material, we used: (1) insoluble rhombohedral-calcite powder (Fig. 4) representing a rheologically hard second phase with isotropic shape, grain diameters of $>150-355$ and $<150 \mu \mathrm{m}$ (most $\sim 20 \mu \mathrm{m}$ ); and (2) graphite (Fig. 5) representing a soft, anisotropic second phase with a grain size of 355 and $150 \mu \mathrm{m}$. We also tested dry compacted crushed ice particles forced together within a cylindrical mould; herein known as 'bubble' ice (Fig. 3a). The bubble ice contained 15 vol.\% porosity defined by air bubbles; as a result of not saturating the pore spaces of crushed and sieved ice particles with liquefied $\mathrm{D}_{2} \mathrm{O}$, prior to annealing the sample. The ice with the insoluble impurity was prepared by dispersing 20 or 40 vol.\% calcite or graphite powder (Table 2), within a crushed and sieved deuterated ice fraction prior to adding $\mathrm{D}_{2} \mathrm{O}$ water. During sample preparation, it was impossible to obtain a completely uniform dispersion of the calcite or graphite between the ice particles with some areas of pure ice preserved during the stirring of the mixture and de-aeration process. In the calcite-ice mixtures, the microstructure is dominated by a bimodal population of irregular-shaped pure ice grains in a matrix of finer irregularly shaped grains (Fig. 4a). In the undeformed finecalcite-ice samples, there is \pm 20 vol.\% of calcite grains (grain diameter $<20 \mu \mathrm{m}$ ) and the matrix ice is dominated by fine irregular grains with a grain size of $\sim 50 \mu \mathrm{m}$ (Fig. $4 \mathrm{~d}$ ).

Two types of in situ experiments were performed:
(1) Cylindrical samples of pure ice and ice with the second phase were deformed in an Instron $100 \mathrm{kN}$ load frame while in situ neutron diffraction measurements were being performed on KOWARI at the Australian Nuclear Science and Technology Organisation (ANSTO; Kirstein and others, 2009). The average length and diameter was $\sim 3.2-4 \times 2.5 \mathrm{~cm}$, respectively, for a length to diameter ratio of $\sim 1.5$ to 1 . Results from four series of deformation experiments with constant displacement rates and starting strain rates of $1 \times 10^{-5} \mathrm{~s}^{-1}$ (named 'fast' experiments) and $2.5 \times 10^{-6} \mathrm{~s}^{-1}$ (named 'medium' experiments) to strains of 10 and $20 \%$ (Table 2). These constant strain rate experiments are part of a series first described by Piazolo and others (2013). Experimental temperature was set to $-7^{\circ} \mathrm{C}$, which is $10^{\circ} \mathrm{C}$ below the melting point for $\mathrm{D}_{2} \mathrm{O}$ ice. These temperature and strain rates, accessible in the laboratory, are close to the rates identified in natural ice $\left(10^{-5}\right.$ to $-10^{-10} \mathrm{~s}^{-1}$; Paterson, 1977; Vaughan, 1993). In fact, the medium strain rate used in the current set of experiments is close to the high-closure rates observed in Antarctic drill holes of 1-1.5 $\times 10^{-6}$ $\mathrm{s}^{-1}$ described by Thwaites and others (1984).

Diffraction peaks of the selected materials were sufficiently different to the $\mathrm{D}_{2} \mathrm{O}$ ice allowing reliable diffraction data analysis (Fig. 1a). Using the intensity of three ice diffraction peaks (002), (100) and (101), we are able to calculate the 


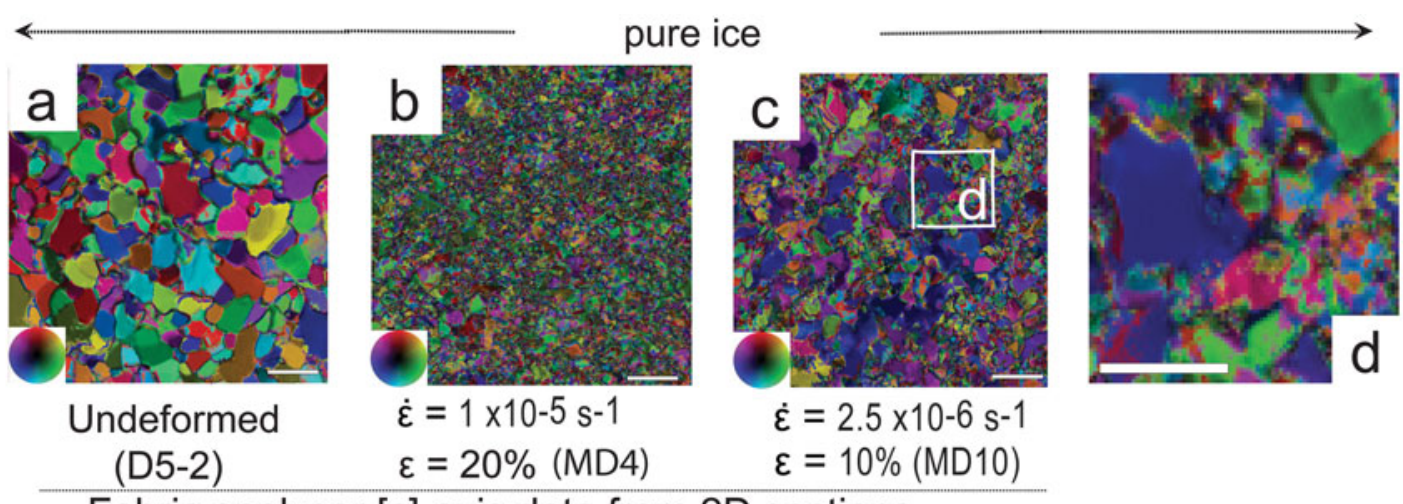

Fabric analyser [c]-axis data from $2 \mathrm{D}$ sections
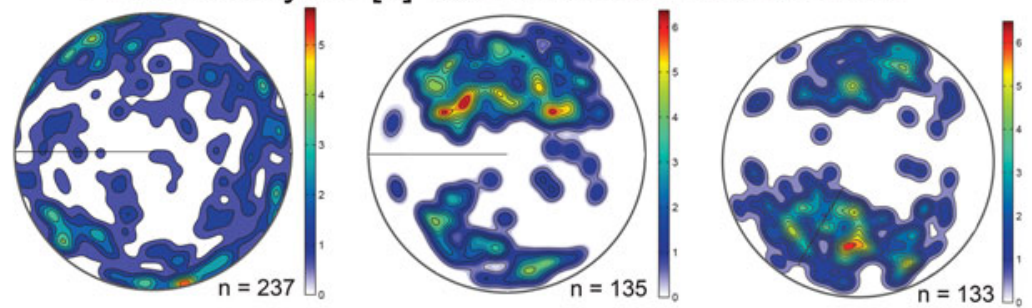

$\dot{\varepsilon}=2.5 \times 10-6 \mathrm{~s}-1$

$\varepsilon=20 \%\left(D 1 \_7\right)$

Neutron diffraction [c]-axis 3D data vertical compression axis
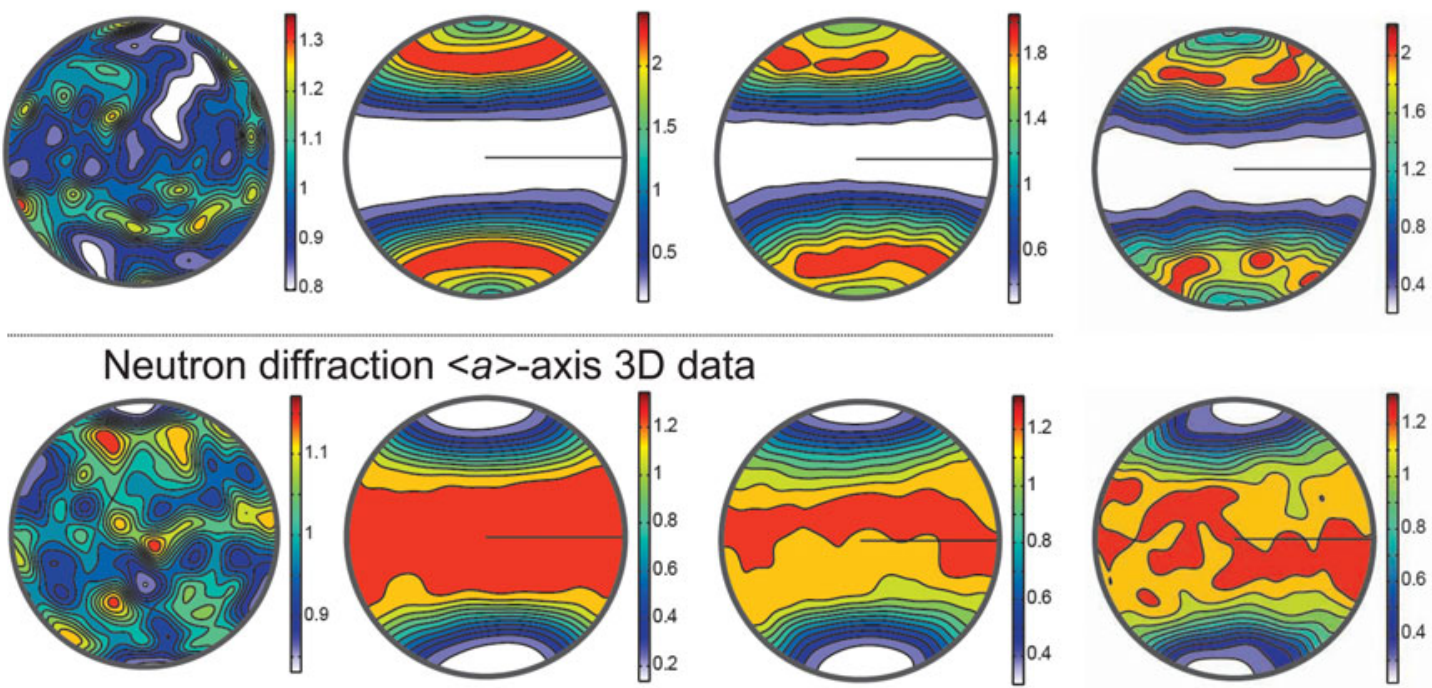

[c]-axis 3D data with compression axis normal to page

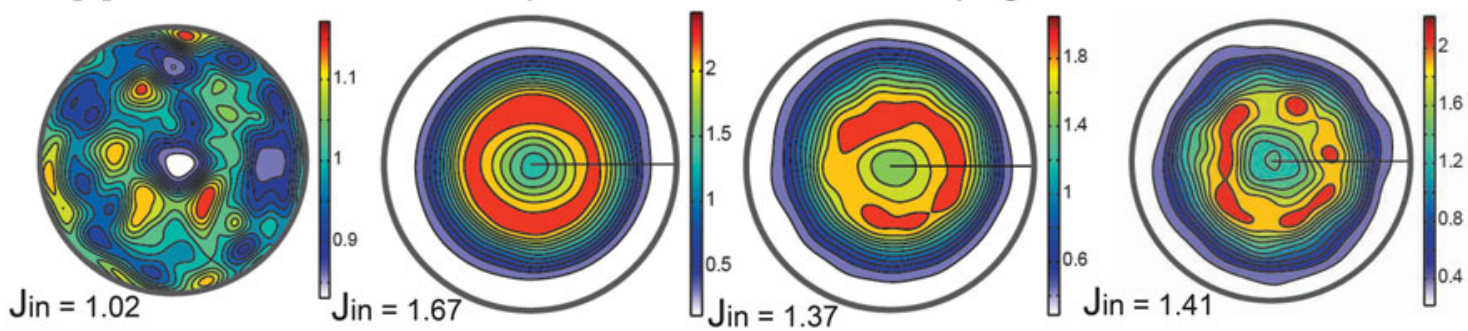

Fig. 2. The starting and final microstructures and $\mathrm{CPO}$ characteristics in deuterated ice samples deformed at $-7^{\circ} \mathrm{C}$. (a-C) Axial distribution analysis (AVA) images showing microstructures before (undeformed) and after a vertical shortening $(\varepsilon)$ of $20 \%$, at strain rates $(\dot{\varepsilon})$ of $1 \times$ $10^{-5} \mathrm{~s}^{-1}$ (fast) and $2.5 \times 10^{-6} \mathrm{~s}^{-1}$ (medium). Bar scale $=1 \mathrm{~mm}=1000 \mu \mathrm{m}$. Inset shows different colours indicating [c]-axis orientation with respect to vertical compression axis. The vertical columns show the $[c]$-axis patterns obtained from the corresponding sample using a G50 fabric analyser, where $n$ is the number of measurements from individual grains plotted as equal-area lower hemisphere projections. Under the fabric analysis, data are the corresponding neutron diffraction [c]- and $<a>$-axis distributions in relationship to a vertical compression direction. The neutron diffraction data are contoured to multiples of uniform distribution. Minima and maxima of density are indicated to the right of each pole figure with the J-index indicated for the overall textures (based on ODF). (d) Enlargement of region in (c) illustrating bimodal grain size distribution. Bar scale $=0.5 \mathrm{~mm}$. (e) Neutron diffraction data obtained from D1_7, no fabric or microstructural data are shown as sample was annealed after texture analysis. 


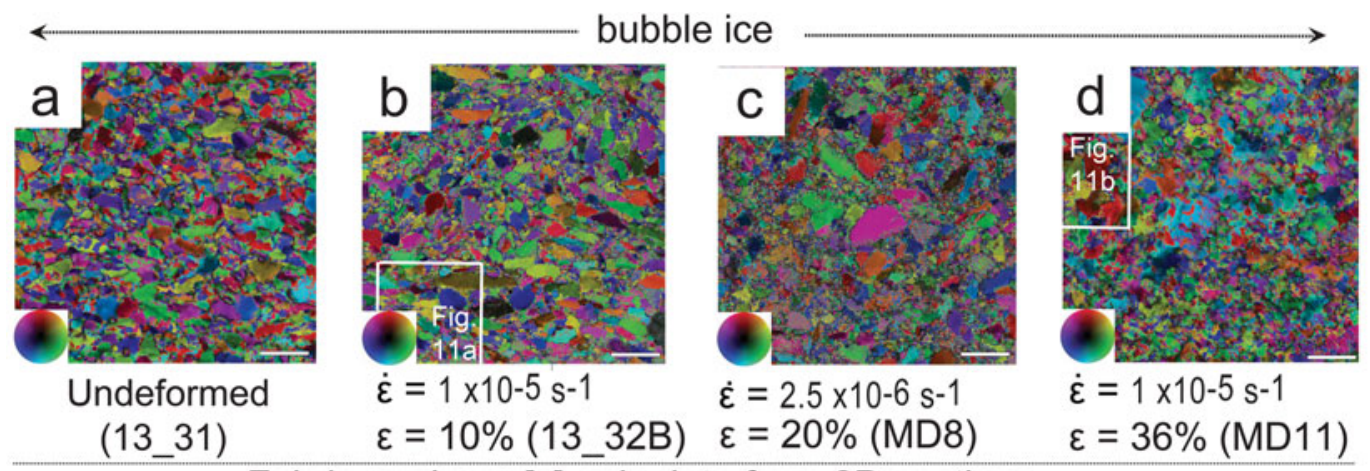

Fabric analyser [c]-axis data from 2D sections

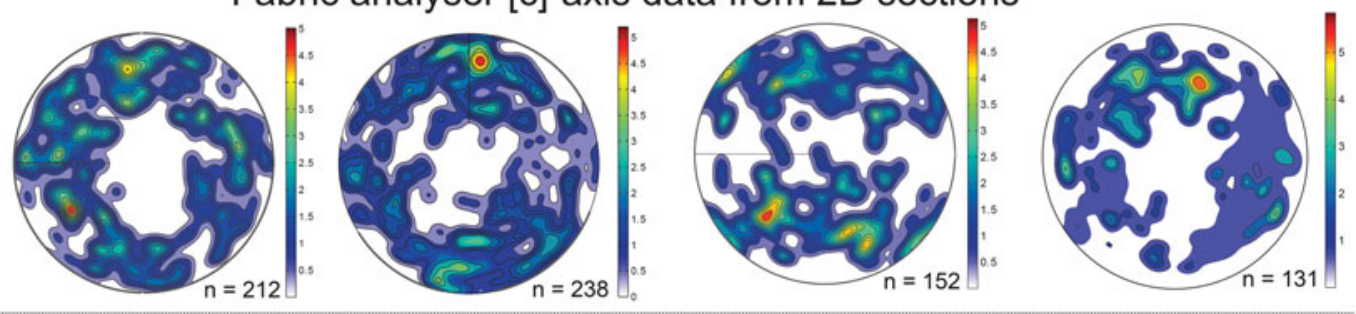

Neutron diffraction [c]-axis 3D data with compression axis vertical
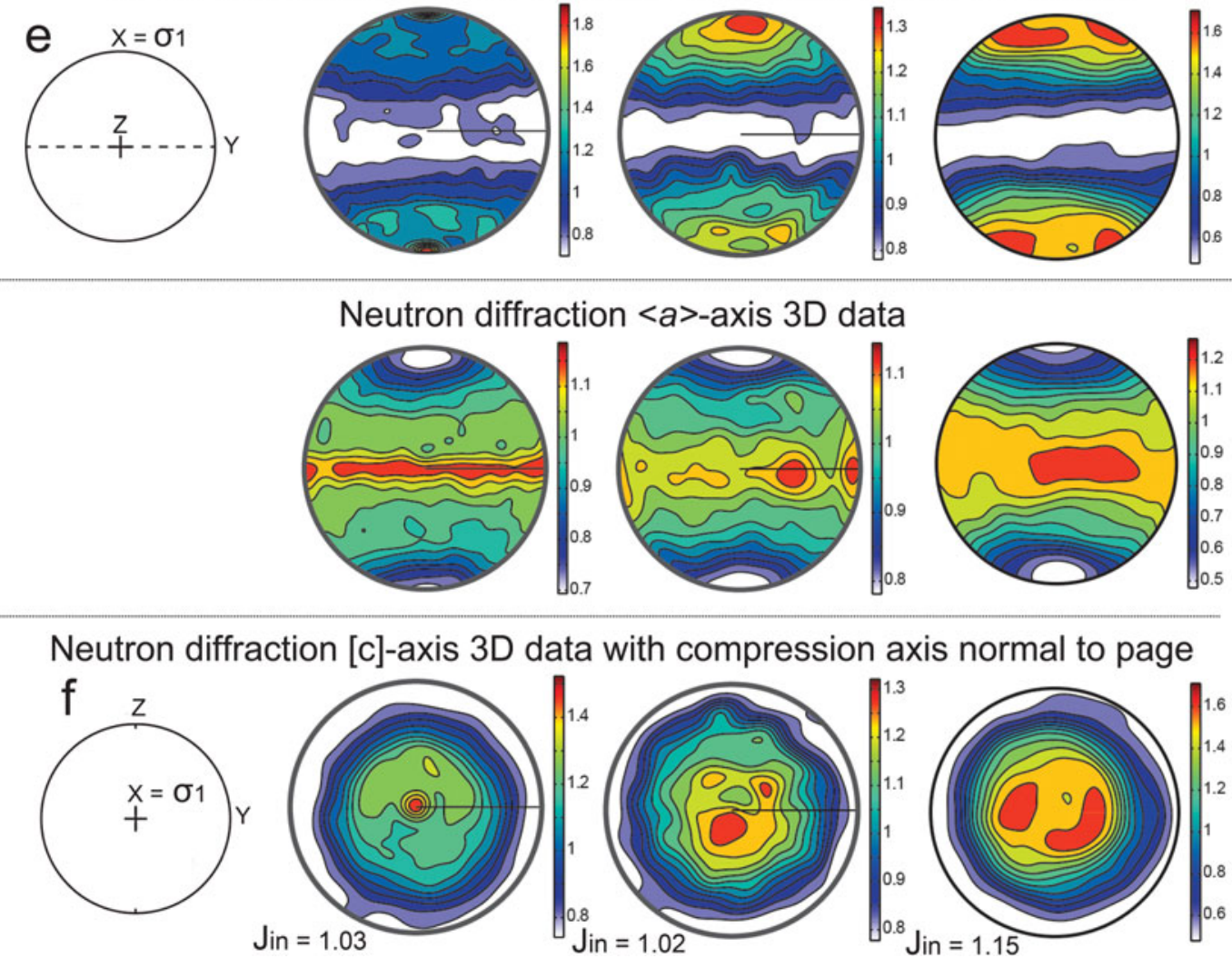

Fig. 3. The starting and final microstructures and $\mathrm{CPO}$ characteristics in deuterated bubble ice samples deformed at $-7^{\circ} \mathrm{C}$. (a-d) Bubble ice samples. (e-f) Geometric coordinates for deformation $X$ (compression axis), $Y=Z$ (lode plane normal directions). (a-d) Axial distribution analysis (AVA) images showing microstructures before (undeformed; a) and after a vertical shortening $(\varepsilon)$ of $10 \%(b, c)$ and $35 \%(d)$, at strain rates $(\dot{\varepsilon})$ of $1 \times 10^{-5} \mathrm{~s}^{-1}$ (fast) and $2.5 \times 10^{-6} \mathrm{~s}^{-1}$ (medium). Bar scale $=1 \mathrm{~mm}=1000 \mu \mathrm{m}$. Inset shows different colours indicating $[c]$-axis orientation with respect to vertical compression axis. The vertical columns show the [c]-axis patterns obtained from the corresponding sample using a G50 fabric analyser, where $n$ is the number of individual grain measurements plotted as equal-area lower hemisphere projections. Under the fabric analysis data are the corresponding neutron diffraction [c]- and $<a>$-axes distributions in relationship to a vertical compression direction. The neutron diffraction data are contoured to multiples of uniform distribution. Minima and maxima of density are indicated to the right of each pole figure with the J-index indicated for the overall textures (based on ODF). The box in (b) indicates location of Figure 11a.The box outline in (d) indicates location of bottom portion of $11 \mathrm{~b}$.

strength of the $\mathrm{CPO}$, change in grain numbers (Fig. 6) and average grain size at small strain intervals (Fig. 7). Partial pole figures from the ice grains were acquired during an experiment, over a range of different directions with respect to the sample by rotating the load frame and sample away from the compression axis ( $\omega$ in Fig. 1a). The range over which $\omega$ was varied was $\pm 35^{\circ}$. In this way, we could continuously monitor the textural and grain size characteristics 

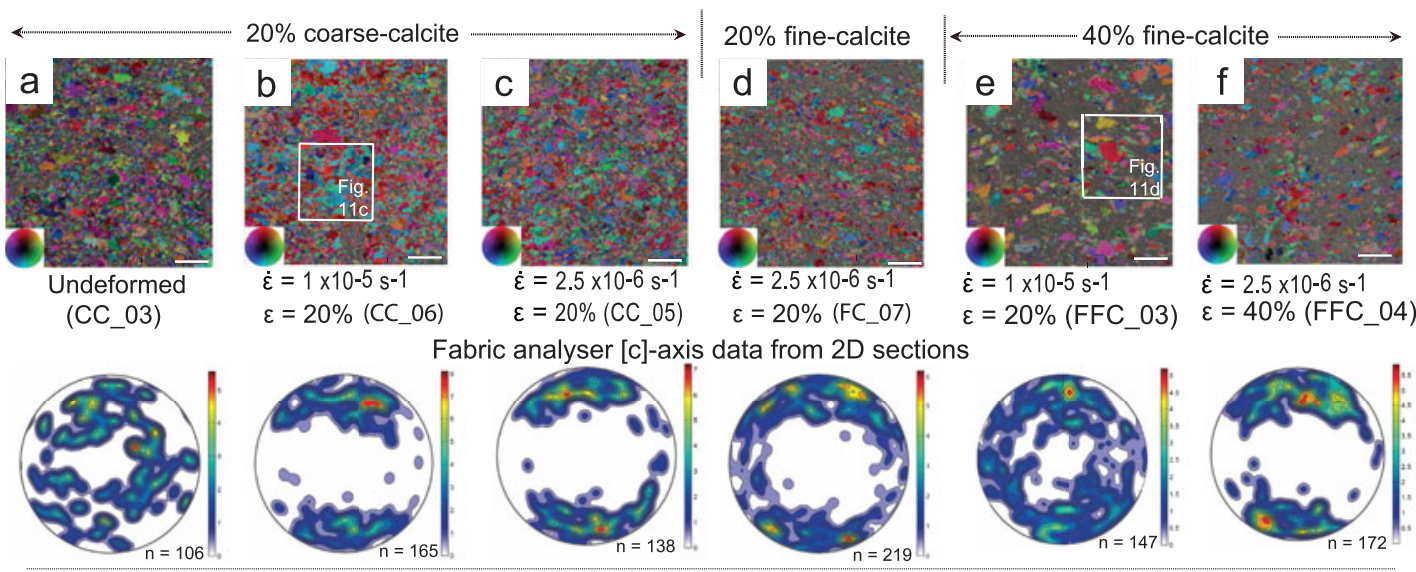

Fabric analyser [c]-axis data from 2D sections
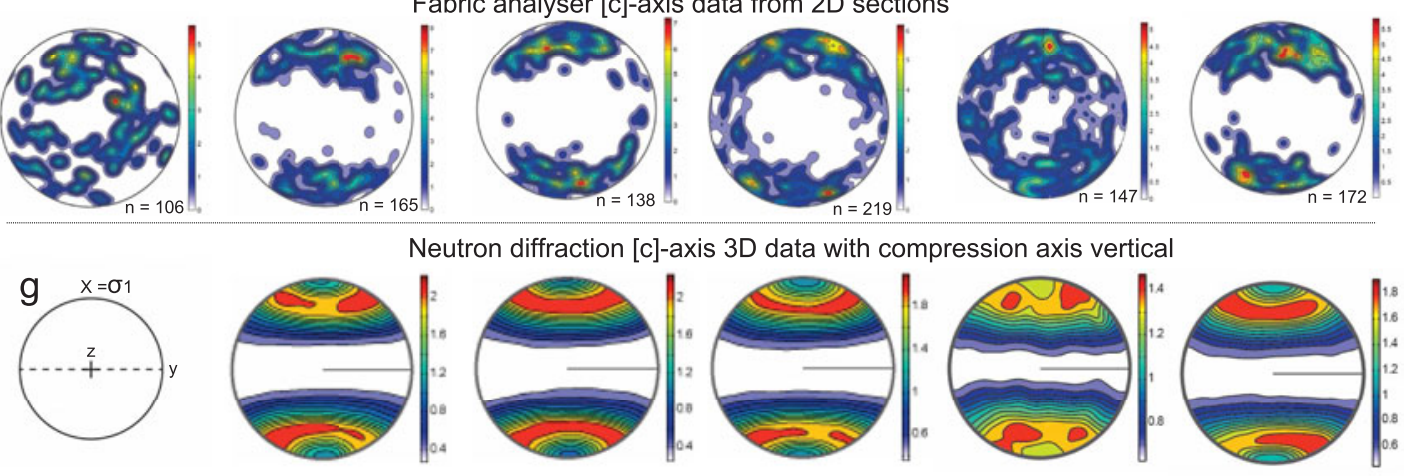

Neutron diffraction $<$ a $>$-axis 3D data
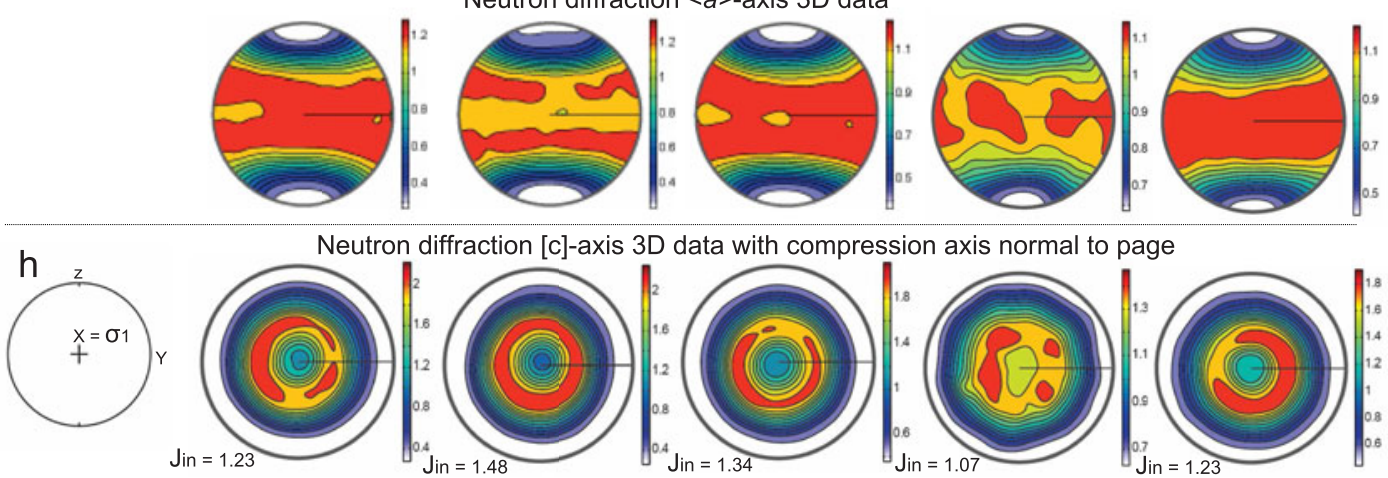

Fig. 4. Microstructure and $\mathrm{CPOs}$ obtained from deformed ice-calcite mixtures. (a-f) Axial distribution analysis (AVA) images showing microstructures before (undeformed) and from samples vertically shortened $(\varepsilon)$. Starting material contained 20 and 40 vol. $\%$ calcite in an ice matrix and were deformed at strain rates $(\dot{\varepsilon})$ of $1 \times 10^{-5} \mathrm{~s}^{-1}$ (fast) and $2.5 \times 10^{-6} \mathrm{~s}^{-1}$ (medium) at $-7^{\circ} \mathrm{C}$. Bar scale $=1 \mathrm{~mm}=1000 \mu \mathrm{m}$. Inset shows different colours indicating [c]-axis orientation with respect to vertical compression axis. The vertical columns show the [c]axis patterns obtained from the corresponding sample using a G50 fabric analyser, where $n$ is the number of measured grains. Under the fabric analysis, data are the corresponding neutron diffraction $[c]-$ and $<a>$-axes distributions with the J-index. Minima and maxima of density are indicated to the right of each pole figure. (g-h) Geometric coordinates for deformation $X$ (compression axis), $Y=Z$ (lode plane normal directions). The boxes in (b) and (e) indicate location of Figs 11c and d.

(Matthies and others, 1987). The full crystallographic orientation data were acquired after the experiment using two-circle Eurelian goniometer for the same three diffraction peaks of ice and (002) peak of graphite. We have reconstructed the orientation distribution function (ODF) from the (002), (100) and (101) ice reflections using the open source toolbox MTEX (http://mtex-toolbox.github.io/; Hielscher and Schaeben, 2008). Calculated pole figures were constructed using a 'de la Vallée Poussin' kernel (half-width $=5^{\circ}$ ). The final CPO strength of ice was quantified using the J-index (Sturcken and Croach, 1963; Bunge, 1982; Mainprice and others, 2014). Before and during data acquisition, samples were kept at temperatures below $-80^{\circ} \mathrm{C}$ to prevent melting and/or annealing.

The average grain size in a sample at different stages during the deformation was obtained from the intensity oscillation of the measured diffraction pattern. An infinite number of grains produce perfect diffraction peaks without any fluctuations, while a small number of grains $(N)$ will give large statistical fluctuations in comparison. The observed diffraction-integrated intensity is a statistical quantity proportional to the neutron beam flux and number of crystallites which are in reflection condition, i.e. comply with Bragg's Law. The number of crystallites $n$ is a random quantity and it is characterized statistically by the expectation $n=N p$ and the std dev. $\sigma^{2}=N p(1-p)$, where $N$ is the total number of crystallites in the sample (or gauge volume) and $p$ is a probability to find a crystallite in the reflection orientation. This probability is determined by neutron optical properties of the instrument, the multiplicity factor of a certain $h k l$-reflection and pole figure density for a given direction and $h k l, p=\mathrm{d} \Omega / ; 4 \pi \cdot M_{h k l} \cdot p f d$. Using the relative std dev., $\frac{\sigma}{n}=(1 / \sqrt{N}) \sqrt{1-p / p}$, eliminates a factor of the neutron flux, and can be used for estimation of the number of grains in the volume provided that the probability is evaluated accurately.

Technically, this approach was realized by (a) dividing the detector in five horizontal slices, (b) integrating of the intensity in each slice, (c) fitting the diffraction peak intensities and (d) finding the average and extracting the std dev. In this way, it was possible to determine $N$ through estimation of the 


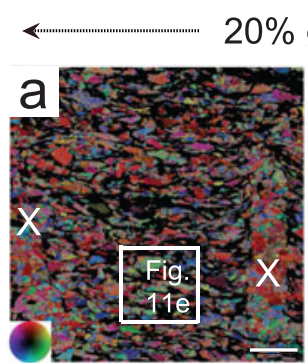

$\dot{\varepsilon}=1 \times 10-5 \mathrm{~s}-1$

$\varepsilon=20 \%\left(G \_02\right)$

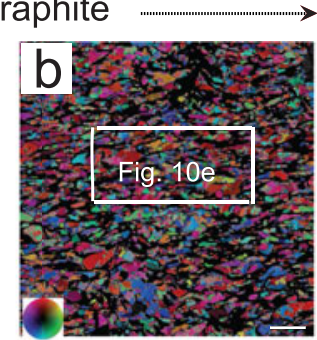

$\dot{\varepsilon}=2.5 \times 10-6 \mathrm{~s}-1$

$\varepsilon=20 \%$ (G_06)

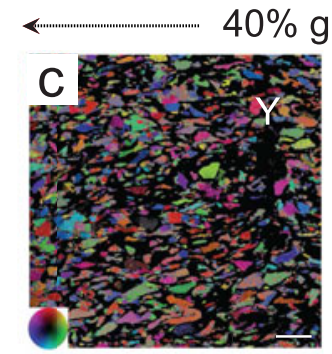

$\dot{\varepsilon}=2.5 \times 10-6 \mathrm{~s}-1$

$\varepsilon=6 \%$ (GG 04)
$40 \%$ graphite

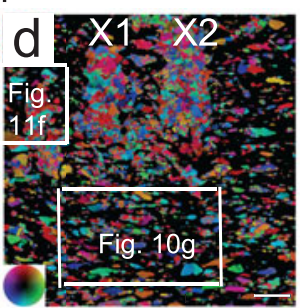

$\dot{\varepsilon}=2.5 \times 10-6 \mathrm{~s}-1$

$\varepsilon=20 \%$ (GG_03)

Fabric analyser [c]-axis data from $2 \mathrm{D}$ sections
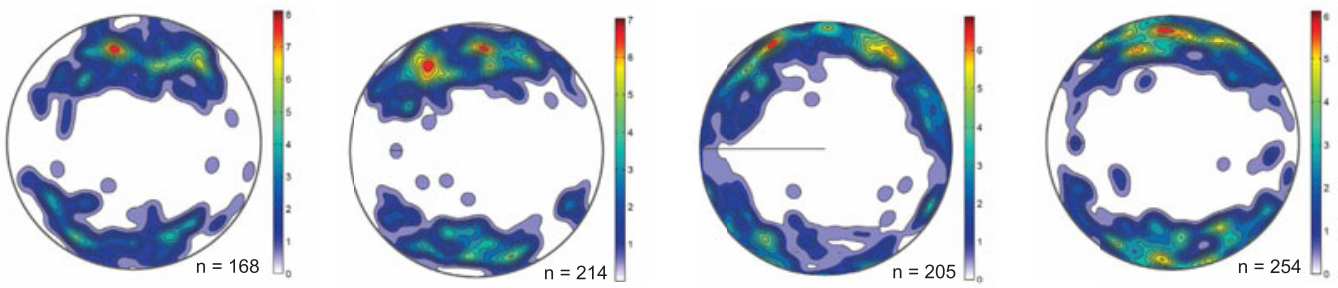

\section{Neutron diffraction ice [c]-axis 3D data}
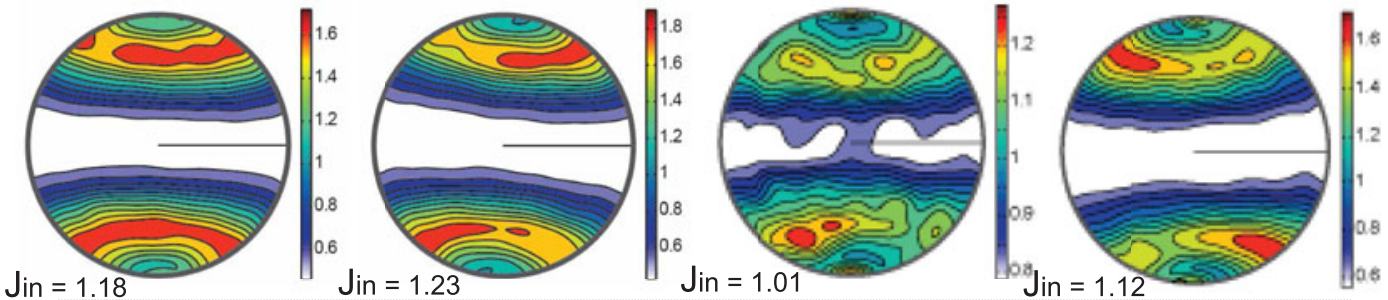

\section{Neutron diffraction ice $<$ a $>$-axis 3D data}
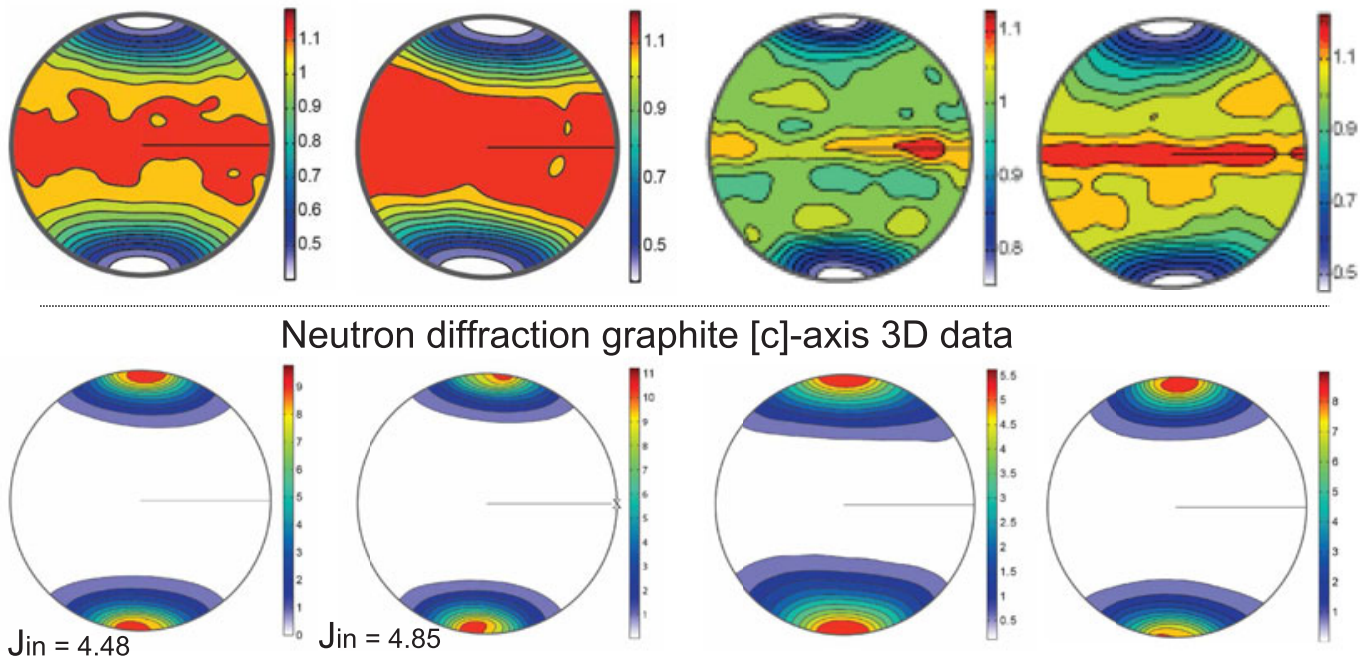

Neutron diffraction graphite [c]-axis 3D data
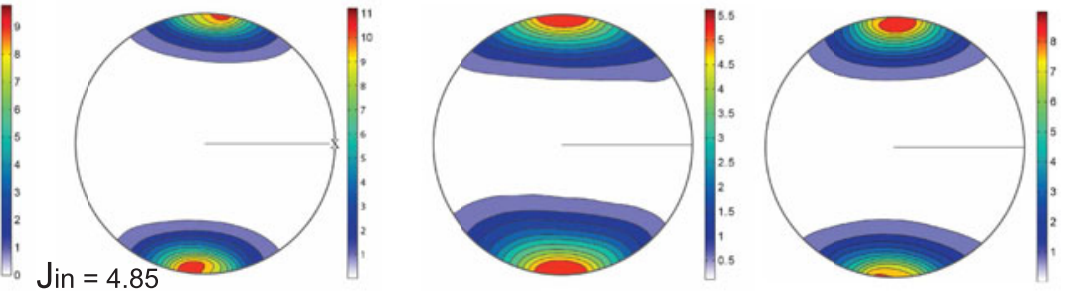

Fig. 5. Final microstructures and $[c]$-axis preferred orientations in ice-graphite samples. The distribution of graphite can be identified as the black areas in the micrographs. (a-d) Sample shortened $20 \%$ at fast strain rate $1 \times 10^{-5} \mathrm{~s}^{-1}$ (fast) and of $2.5 \times 10^{-6} \mathrm{~s}^{-1}$ (medium) at $-7^{\circ} \mathrm{C}$. Bar scale $=1 \mathrm{~mm}=1000 \mu \mathrm{m} . X$ and $Y$ are concentrations of ice and graphite, respectively, left behind during the sample preparation process. The vertical columns show the [c]-axis patterns obtained from the corresponding sample using a G50 fabric analyser, where $n$ is the number of measured grains. The corresponding neutron diffraction $<a>$-axes and $[c]$-axes distribution in ice and $[c]$ distributions in graphite with the J-index, viewed with the compression axis parallel to the edge of the page. Minima and maxima of density are indicated to the right of each pole figure. The boxes in (a), (b) and (d) indicate location of Figs 11e, 10e, 11f, 10g.

relative std dev. at each of the $1^{\circ}$ steps and for each diffraction peak. Moreover, the number of grains was corrected by taking into account the developing texture based on information in the partial pole figure. At the end, individual grain number evaluated for particular angular position and $h \mathrm{kl}$ reflections were averaged to produce an estimate of the numbers of grains in a sample and consequently the mean grain size. Statistical analysis showed that mean grain size estimate is accurate within $5 \%$ std dev. By using this technique, we can establish the number of grains in a given volume of ice at any stage during the deformation. For example, in the initial pure ice samples MD10 and MD04 (Fig. 6), the number of grains were $46300 \pm 400$ and 53 $200 \pm 400$, respectively, a comparable result, as the samples were produced under almost identical initial conditions. As the deformation proceeds, this number increases as 


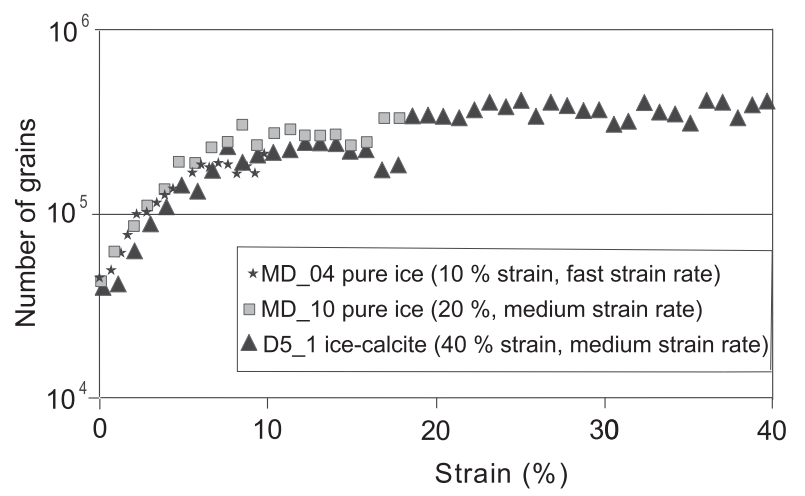

Fig. 6. Examples of grain number evaluation at different stages during the $-7^{\circ} \mathrm{C}$ deformation of the ice samples. These numbers account for volume changes during deformation with an error in the grain number determination of approximately \pm 400 (see 'Methods' and 'Grain size evolution' sections for further descriptions).

new grains are nucleated, depending on deformation conditions (temperature, strain and strain rate). A strain rate dependence is weakly pronounced, the grain number evolution as a function of the compressive deformation is more apparent; with the number of grains stabilizing after $10 \%$ shortening reaching a steady-state condition (Fig. 6). This is valid for pure ice samples (e.g. MD04 or MD10) and twophase samples (e.g. D5_1).
In addition, we examined the starting and final microstructures in $\leq 250 \mu$ m-thick thin sections for all the sample types using a G50 fabric analyser (Wilson and others, 2003; Wilson and Peternell, 2011). Thin sections were cut parallel to the compression direction, and all fabric analysis data are represented with the compression direction oriented in a horizontal north-south orientation (Figs 3e, f). We used FAME software (Fabric Analyser Microstructural Evaluation; Hammes and Peternell, 2016) to process the fabric analyser data and crossed-polarized photographs to determine the position of calcite and graphite grains.

(2) The second set of experiments involved in situ co-axial plane strain 2-D experiments (Figs 1b, c) with time-lapse observations through thin wafers cut from the cylindrical samples. A $250 \mu \mathrm{m}$-thick $\sim 15 \times 25 \mathrm{~mm}$ sized wafer was placed in a plane strain, pure shear deformation press under an automatic fabric analyser (Wilson and others, 2003; Peternell and others, 2011 for further discussion). Differential strain rates were achieved by changing the rate at which the piston was advanced into the sample (Table 2). An area containing equiaxed ice grains, $5 \times 5$ $\mathrm{mm}$ or $1000 \times 1000$ pixels, was located within the 250 $\mu \mathrm{m}$-thick wafer and a record of microstructural evolution with a spatial resolution of $5 \mu \mathrm{m} /$ pixel was recorded every $4 \mathrm{~min}$ (Peternell and Wilson, 2016). The single-frame measurements from the fabric analyser were combined with results from a FAME analysis (Hammes and Peternell, 2016).

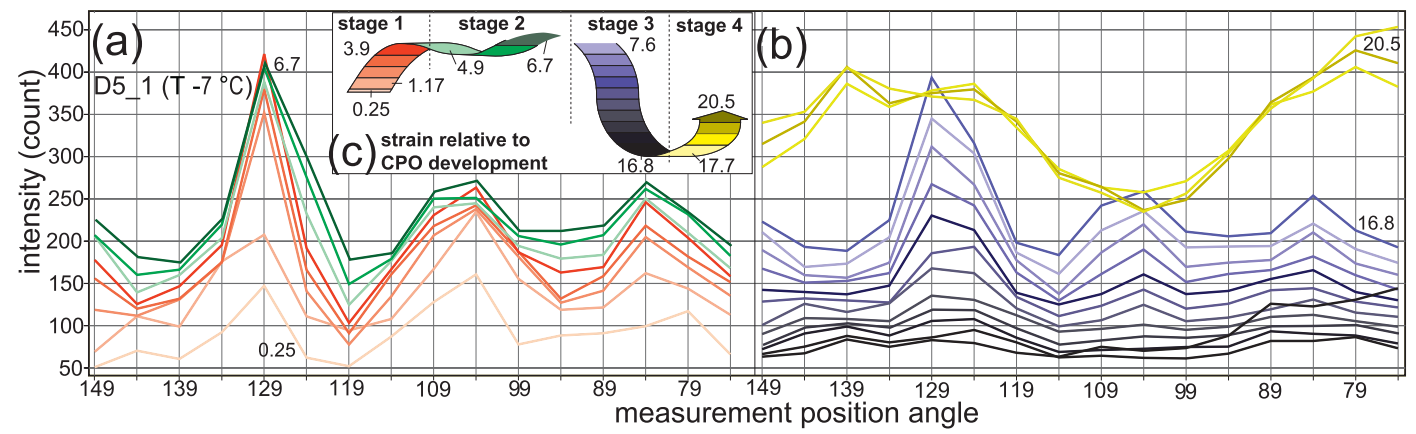

(d)

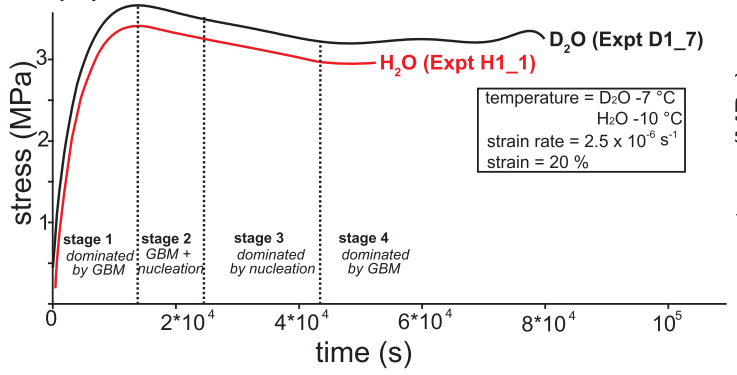

(e)

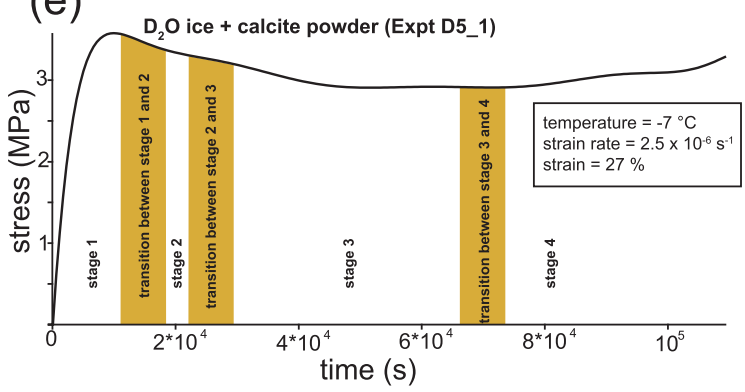

Fig. 7. Interpretation of microstructural evolution from pole figure measurements derived from neutron diffraction measurements during a 3-D uniaxial deformation at $-7^{\circ} \mathrm{C}$ and a medium strain rate of $2.5 \times 10^{-6} \mathrm{~s}^{-1}$ (modified from Wilson and others, 2014). (a-b) Successive intensity profiles in an ice-calcite sample (D5_1) scaled by counts for the [002] axis relative to position angle, or the number of neutrons reflected off the (002) crystallographic plane at different finite strains. Colour code shows intensity with respect to increasing strain from (a) $0.25-3.9 \%$ shortening (light red-reddish to brown colours) and 4.9-6.7\% shortening (light green to dark green); (b) 7.6-16.8\% shortening (light blue to grey-blue to black) and 17.7-20.5\% shortening (light yellow to yellow-green). The inset (c) shows relative slope of the finite strain path with change in the intensity profile with positive slope $=$ increase and negative slope $=$ decrease. The relative height differences in the finite strain path represent the magnitude of the strain increments for each stage. (d) Creep curves that compare pure $\mathrm{D}_{2} \mathrm{O}$ (Experiment D1_7) with $\mathrm{H}_{2} \mathrm{O}$ ice (Experiment $\mathrm{H}_{1} \_$; ; after Wilson and others, 2014). (e) Creep curve for $\mathrm{D}_{2} \mathrm{O}$ ice and coarse calcite (D5_1). The microstructural stages are distinguished on the basis of intensity changes during neutron diffraction and optical observations from comparable 2-D and 3-D experiments. 
During this investigation, the accuracy of some individual [c]-axis measurements in the two-phase ice, obtained while using 'Investigator' software (Wilson and others, 2007), was generally interfered with by the presence of either fine calcite or a non-uniformity in the optical continuity of the ice (e.g. undulose extinction and/or subgrains). Such data were rejected on the basis of their geometrical and retardation quality values (Peternell and others, 2009), which meant $[c]$-axis determinations in pole figure diagrams (Figs $2-5)$ are $<200$ points, even though up to $\sim 500$ grains were selected (see explanation in Appendix).

\section{MECHANICAL DATA}

The nature of the creep and stress-strain paths is illustrated in Figures $7 d$, e, 8. In the 2-D experiments (Fig. 8a), three strain rate scenarios were used, namely: (1) constant displacement rates with starting strain rates of $2.5 \times 10^{-6} \mathrm{~s}^{-1}$, which is the same as the corresponding medium $3-\mathrm{D}$ experiments (Table 2; Fig. 8c); (2) short interval strain rate cycles where strain rates varied from $\sim 1.9 \times 10^{-6}$ to $3.1 \times 10^{-6} \mathrm{~s}^{-1}$, accompanied by incremental strain variations of $0.14-0.21 \%$. Overall the average path was identical to a medium, $2.5 \times$ $10^{-6} \mathrm{~s}^{-1}$ strain rate; (3) long interval strain rate cycles of $\sim 2.5 \times 10^{-6}$ to $3.1 \times 10^{-6} \mathrm{~s}^{-1}$ and strains varied between 2.9 and $5.5 \%$. The fast 3-D experiments (Fig. 8b) have starting strain rates of $1 \times 10^{-5} \mathrm{~s}^{-1}$.

In the 3-D experiments, the load $(F)$ was measured at small time steps (<2 s) on a fixed grip (Fig. 1a) and then recalculated to stress $(\sigma)$ using the relationship:

$$
\sigma=(F-f) / A
$$

where $f$ is the friction, and $A$ is the increasing area of the cylinder, assuming the uniform strain and near constant sample volume. Sublimation of ice on both grips is considered to be minimal with a friction $(f)$ of $\sim 0.1 \mathrm{kN}$ between the grips and the aluminium cooler (Fig. 1a). The volume change by extrusion of air in the dry compacted or 'bubble' ice could not be calculated. The calculated sample diameter was identical to the sample diameter measured after the experiment. As axial strain rate increases with strain, strain rate was separately calculated for each time step together with the average grain size in a sample at different stages during the deformation.

A subset of our stress-strain data obtained in the 3-D experiments is plotted in Figures $8 \mathrm{~b}, \mathrm{c}$. The pure ice and calcite-bearing ice show similar stress-strain relationships throughout the experiment (Figs 8b, c). They first undergo strain hardening, reaching the peak stress at $\sim 2 \%$ strain, which is then followed by strain weakening, and a period of nominal constant stress interpreted as mechanical steady state. The mechanical steady state or flow stress values $\left(\sigma_{\mathrm{ss}}\right)$ and peak stress values of ice with calcite are greater than those of the pure and bubble-rich ice (Figs 8b, c). The bubble ice is the weakest material, first undergoing strain hardening then slowly hardens at a steady state well below the flow stress of the pure ice.

The samples with fine-grained calcite exhibit a higher peak (3.5 MPa at $\dot{\varepsilon}=1 \times 10^{-5} \mathrm{~s}^{-1} ; 2.8 \mathrm{MPa}$ at $\dot{\varepsilon}=2.5 \times$ $\left.10^{-6} \mathrm{~s}^{-1}\right)$ and steady-state stresses than the coarse-grained calcite sample. Also, ice with calcite reaches mechanical steady state at higher strains than the pure material (Figs $8 \mathrm{~b}, \mathrm{c})$ and may progressively harden (Fig. 7e). The effects

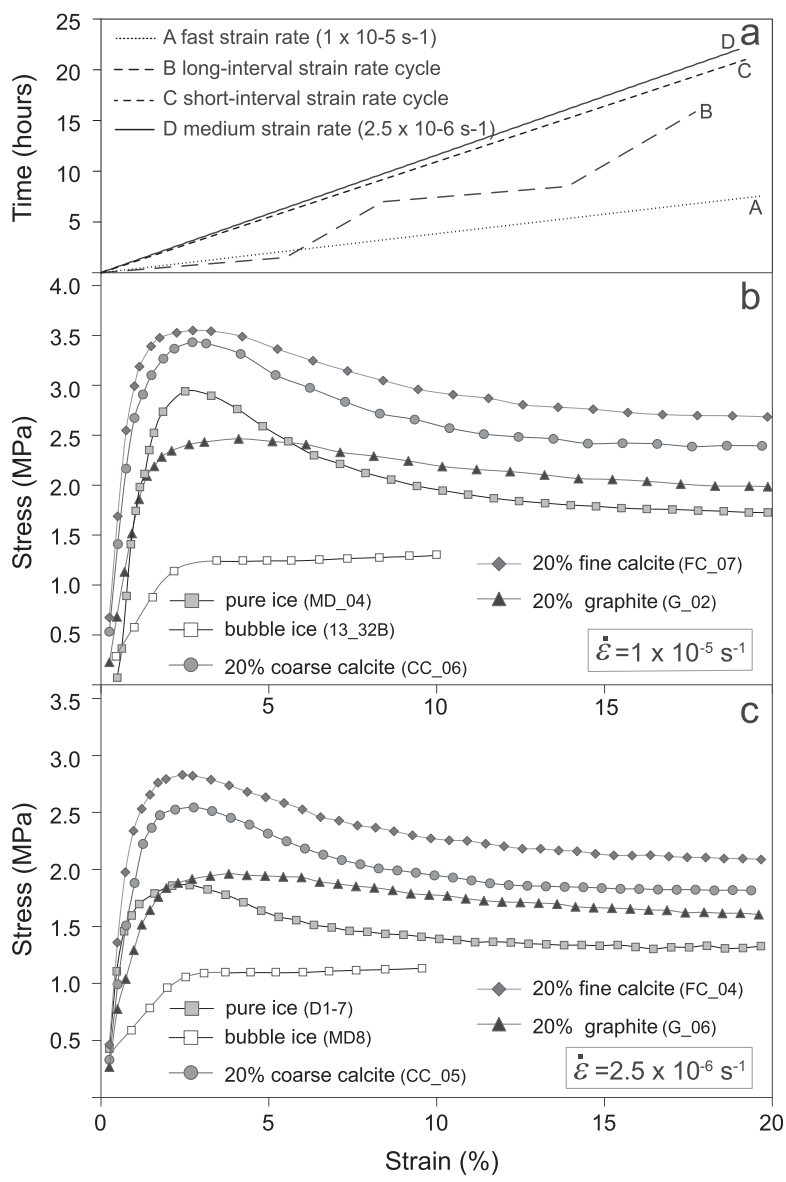

Fig. 8. Nature of the creep and stress-strain relationships for a subset of the pure ice and ice mixture experiments. (a) Four creep curves showing strain against time under particular strain rates in 2-D fabric analyser experiments. Lines $A=1 \times 10^{-5} \mathrm{~s}^{-1}$ represents a fast strain rate; Line $\mathrm{B}=$ long interval strain rate cycle from $1.02 \times 10^{-5}$ to $1.45 \times 10^{-6} \mathrm{~s}^{-1}$ (see Peternell and Wilson, 2016). The sigmoidal shape of line B represents long interval strain rate cycles where strain rates were $\sim 2.5 \times 10^{-6}$ to $3.1 \times 10^{-6} \mathrm{~s}^{-1}$ and strains varied between 2.9 and $5.5 \%$. Line $\mathrm{C}=$ short interval strain rate cycles where strain rates varied from $\sim 1.9 \times 10^{-6}$ to $3.1 \times 10^{-6} \mathrm{~s}^{-1}$, accompanied by strain variations of $0.14-0.21 \%$. Line $D=2.5 \times$ $10^{-6} \mathrm{~s}^{-1}$ representing a medium strain rate. (b-c) Instantaneous stress plotted against strain for 3 -D experiments conducted at constant strain rates of (b) $1 \times 10^{-5} \mathrm{~s}^{-1}$ (fast), (c) $2.5 \times 10^{-6} \mathrm{~s}^{-1}$ (medium) (modified after Cyprych and others, 2016). The variation in stress for each curve is $< \pm 0.40 \mathrm{MPa}$.

of adding fine-grained calcite vs coarse calcite not only increases the hardening of the ice but provides additional nucleation points for recrystallization (Figs $4 d-f$ ). The stress-strain relationship of graphite-bearing ice does not show a distinct stress drop after the peak stress is reached. After $\sim 3 \%$ strain, stresses decrease steadily throughout the experiment (Fig. 8). The peak stress is comparable to that of pure ice at a strain rate of $2.5 \times 10^{-6} \mathrm{~s}^{-1}$. At a strain rate of $1 \times 10^{-5} \mathrm{~s}^{-1}$, the peak stress is lower than that of pure ice.

A subset of our experimental results were analysed by Cyprych and others (2016) in terms of a constitutive equation related to a dislocation creep flow law in the form:

$$
\dot{\varepsilon}_{\mathrm{ss}}=A \sigma_{\mathrm{ss}}^{n} \mathrm{e}^{-\left(\frac{Q}{R T}\right)},
$$

where $\dot{\varepsilon}_{\mathrm{ss}}-$ calculated steady-state strain rate, $A$ - material constant, $\sigma_{\mathrm{ss}}-$ steady-state stress, $n-$ stress exponent, $Q-$ 
Constant strain rate

$(2.5 \times 10-6 s-1)$ pure ice

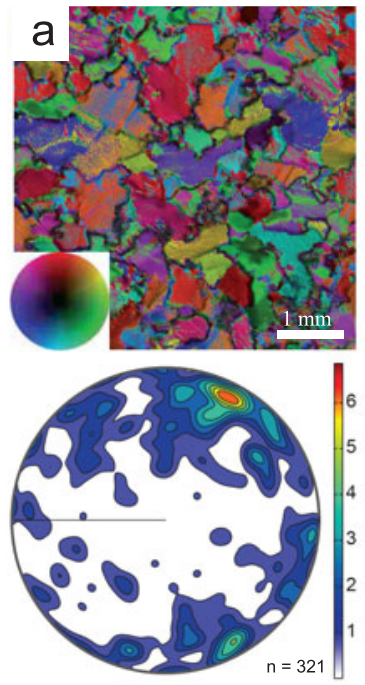

Short-interval strain rate cycle - pure ice
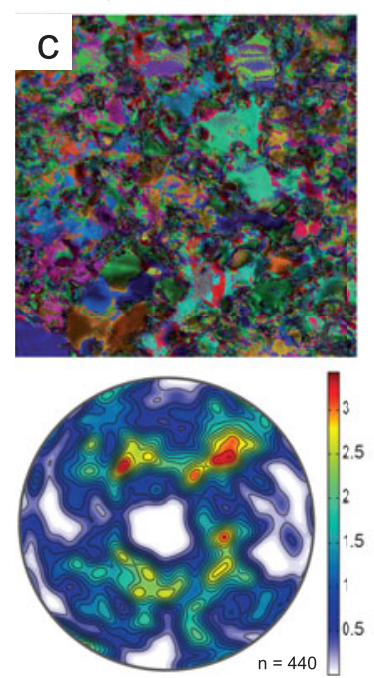

Long-interval strain rate cycle - pure ice
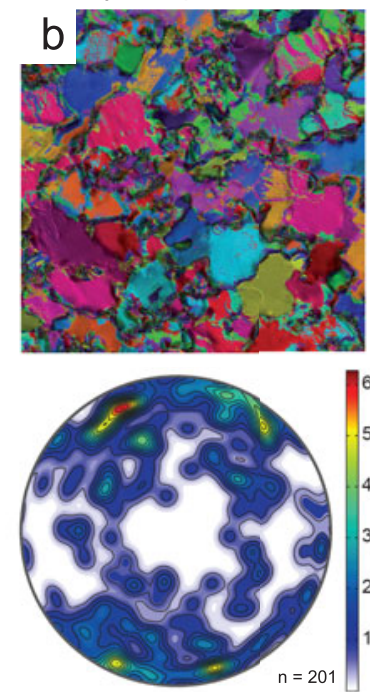

Short-interval strain rate cycle - ice and $20 \%$ calcite
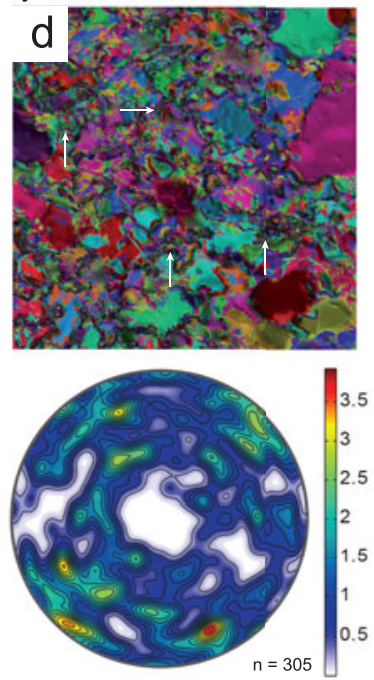

Fig. 9. Microstructure and CPOs obtained after 20\% shortening in 2D fabric analyser experiments with variable strain rates $(\dot{\varepsilon})$ at $-7 \mathrm{C}$. Minima and maxima of density are indicated to the right of each pole figure. (a) Pure ice after a constant $\dot{\varepsilon}=2.5 \times 10^{-6} \mathrm{~s}^{-1}$ deformation. Bar scale $=1 \mathrm{~mm}$ and inset shows different colours indicating $[c]$-axis orientation with respect to vertical compression axis that applies to all images. (b) Pure ice deformed as cycles of fast $\left(\dot{\varepsilon}=10.2 \times 10^{-6} \mathrm{~s}^{-1}\right)$ involving increments of $0.3 \%$ shortening, followed by relatively slower cycle $\left(\dot{\varepsilon}=1.45 \times 10^{-6} \mathrm{~s}^{-1}\right)$ involving $0.04 \%$ shortening increments. (c) Deformed pure ice and (d) ice with coarse calcite after numerous short cycles of 'fast' $(\dot{\varepsilon}=2.8 \times$ $\left.10^{-6} \mathrm{~s}^{-1}\right)$ involving $0.07 \%$ shortening followed by slow $(\dot{\varepsilon}=1.9 \times$ $10^{-6} \mathrm{~s}^{-1}$ ) involving $0.005 \%$ shortening increments. In (c) the highly irregular grains display strong undulose extinction and in (d) many grain boundaries are pinned by the calcite as highlighted by white arrows.

activation energy, $R$ - gas constant and $T$ - temperature (Durham and others, 1992). The calculated $n$ for the pure ice is close to 3.3, while for calcite- and graphite-bearing ice equals 5-5.6 and 6.6, respectively. These values are comparable to the values reported by Middleton and others (2017) in fluorite-ice mixtures. There were insignificant data to obtain a steady state and record the significant volume changes during the deformation of the bubble ice and no representative flow law was obtained.

\section{MICROSTRUCTURAL EVOLUTION}

\subsection{Pure and dry compacted ice}

The pure ice, after a fast 3-D deformation (Fig. 2b), displays prominent intracrystalline deformation features such as wavy (0001) undulose extinction and subgrain boundaries $\left(<10^{\circ}\right)$ in the majority of grains. The matrix is dominated by small grains $(\sim 50 \mu \mathrm{m})$ between which are larger grains $(\sim 200 \mu \mathrm{m})$ with undulose extinction and deformation bands. Similar microstructures (Fig. 9) have also been observed in the final stages of 2-D in situ short-interval experiments involving fast vs medium strain rates (Peternell and Wilson, 2016).

Following a medium strain rate deformation of pure ice (Fig. 2c), there was a bimodal population of small $(\sim 100$ $\mu \mathrm{m})$ irregular-shaped new grains between larger (200-300 $\mu \mathrm{m})$ grains (Figs $2 \mathrm{~d}, 10 \mathrm{c}$ ). All of the larger grains have interlobate shapes, irregular boundaries and minor distortions in the form of gentle undulose extinction, which transition into subgrains and new grains (Figs 2c, d, 10b, c). These observations are comparable to the 2-D in situ constant medium strain rate experiments (Fig. 9a) described by Peternell and Wilson (2016); where the same (Fig. 2a) starting material was used, with a grain size of $\sim 300 \mu \mathrm{m}$ and a CPO very close to random. After deformation, the ice had its mean grain size of $\sim 230 \pm 12 \mu \mathrm{m}$, during a stage of new grain nucleation accompanied by grain boundary migration. If strain rate is varied (Fig. 8a), over long intervals (Fig. 9b), grain size reduction is achieved by a combination of intracrystalline deformation followed by grain boundary migration to produce microstructures (Fig. 9b) identical to the 3-D experiments (Figs 2c, d).

During a fast deformation of the bubble ice (Fig. 3b), the pores disappear producing a more coherent matrix dominated by irregular-shaped grains, and displaying significant undulose extinction and subgrain development. The grains are markedly elongate perpendicular to the shortening direction with highly serrated and wavy grain boundaries (Fig. 11a). The smallest air bubbles are located on grain boundaries and remain spherical. After $10 \%$ shortening during a medium strain rate deformation (Fig. 3c), the grain size distribution is bimodal, dominated by small equant grains and larger elongate grains with undulose extinction (Fig. 10a). Bubbles have a pinning effect and appear to restrict the onset of grain boundary migration. A fast deformation with $35 \%$ shortening produces irregular grains with a lobate interlocking grain microstructure (Fig. 11b).

\subsection{Calcite-ice mixtures}

After $20 \%$ shortening in the fast 3-D experiments, the grain size of ice containing the coarser calcite fraction decreases (Table 2; Fig. 4b). The ice is dominated by irregular large $(\sim 200 \mu \mathrm{m})$ and small $(100 \mu \mathrm{m})$ grains with highly lobate grain boundaries that are pinned by films of calcite (e.g. see white arrows in Fig. 11c). Intracrystalline distortion, undulose extinction and subgrains occur in all samples with 20 vol.\% calcite (Figs 4b, c). During a slower strain rate (Fig. 4c), grain boundaries are smoother and the presence of intracrystalline deformation features is considerably reduced. In the fine-calcite mixtures (Fig. 4d), the final grain sizes are significantly reduced $(\sim 50-250 \mu \mathrm{m})$; there is a heterogeneous distribution of larger single crystals and significant pinning of grain boundaries is observed. As the 
volume of fine calcite is increased (40 vol.\%), the fraction of smaller grains with highly curved or lobate grain boundaries increases and dominates the matrix (Figs 4e, f, 11d). If the initial dispersion of calcite is uneven, then there may be isolated ice grains with diameters of $\sim 500 \mu \mathrm{m}$ (Figs $4 \mathrm{e}, \mathrm{f}$ ).

In the 2-D short-interval strain rate experiments, the final microstructure in the pure ice (Fig. 9c) and ice with calcite (Fig. 9d) is similar to the 3-D medium strain rate experiments (Figs 4c, d); with irregular-shaped ice grains embedded in a matrix of finer $(\sim 120 \mu \mathrm{m})$ ice (Fig. 9c) or interspersed between and pinned by the calcite (Fig. 9d). The initial ice in both the 2-D and 3-D experiments had a close to random $\mathrm{CPO}$; however, there were contrasting $\mathrm{CPO}$ at the end of the experiments (see discussion on preferred orientation development).

\subsection{Graphite-ice mixtures}

During sample preparation, which involves compaction to expel excess water, a weak preferred alignment of the graphite basal planes developed perpendicular to the compression direction. Locally, graphite platelets were congealing to form larger aggregates. Irregular-shaped ice crystals with variable grain sizes $(<1000 \mu \mathrm{m})$ were located between these graphite grains and aggregates. At the conclusion of a fast strain rate experiment involving 20\% shortening (Fig. 5a), with 20 vol. $\%$ graphite, the graphite flakes and aggregates were flattened around ovoid-shaped ice particles. Many of the ice crystals in the matrix displayed optical evidence of intracrystalline deformation features such as undulose extinction and deformation band development (Fig. 11e). Whereas, in the localized channels of pure ice (area marked $X 1, X 2$ in Fig. 10f), there are lobate-shaped coarser ice grains.

After $6 \%$ shortening at a medium strain rate (Fig. $5 \mathrm{C}$ ), with 40 vol. \% of graphite flakes, there was the development of a clear shape alignment of the isolated ovoid-shaped ice particles within an aligned matrix of graphite flakes. All ice particles displayed marked intracrystalline deformation features (Fig. 5c). At the conclusion of 20\% shortening (Fig. 5d), the ice microstructure was dominated by a wavy pattern of the graphite (001) basal planes around lensoidal-shaped recrystallized ice aggregates. The graphite then defines interconnected layers of a weak material (Figs 10f, g) between the harder ice aggregates.

Within the graphite-bearing samples, there were also inherited channels of pure ice ( $X$ in Figs $5 \mathrm{a}, \mathrm{d}$ ) and graphite ( $Y$ in Fig. 5c), which were artefacts developed during sample preparation. These were produced during the removal of a mixing needle, which left a $1 \mathrm{~mm}$-wide void that became infilled by fine grains of either graphite or $\mathrm{D}_{2} \mathrm{O}$ ice. These channels were aligned at a high angle to the initially weak graphite CPO and sub-parallel to the subsequent compression direction. During deformation, these dark seams of graphite ( $Y$ in Fig. 5c) allow the collapse of the adjacent dispersed ice-graphite mixtures producing undulations within the sample. Similarly, the ice channels, now infilled with larger recrystallized ice grains (Figs 5a, d, 10f) are more robust and the matrix flows around these to produce undulations in the shape distribution of the adjacent ice lenses and graphite alignment. Hence the internal geometry in these mechanically anisotropic materials when compressed depended upon the degree of anisotropy of the material and the orientation to the principle compression direction. The mineral migration observed in response to stress gradients established during the development of these structures results in discrete zones of weaknesses and hence the deformation in these samples is locally inhomogeneous.

\section{GRAIN SIZE EVOLUTION}

Comparisons of optically derived grain sizes, analysed after the termination of deformation (Table 2), are comparable to those obtained using the neutron diffraction analysis method. This is reflected in the correlation between average grain size evolution and strain during progressive deformation (Fig. 11) and is indicative that we are in a grain size-insensitive regime (De Bresser and others, 1998). All materials of differing initial grain sizes (Table 2) show a clear tendency to decrease with increasing strain with the establishment of a stabilized grain size (Fig. 12). This decrease in grain size is particularly marked after $\sim 3 \%$ strain which corresponds with a drop in peak stress (Fig. 8c). During the initial 5\% shortening in all two-phase samples, particle pinning leads to an increase of intracrystalline distortions within the ice (Fig. 11) and restricts grain boundary migration.

The microstructure produced in pure ice and calcitebearing ice during a medium strain rate neutron diffraction experiment has a similar grain size evolution (ND in Fig. 12). In pure ice, there is a small decrease in grain size in the first $1 \%$ strain, followed by further grain size reduction between 1 and 3\% strain. Between 3 and 10\% strain, grain size decreases gradually with minor fluctuations and between 10 and $15 \%$ strain stays the same. After $15 \%$ strain, grain size again decreases gradually. Grain size of ice in the coarse-grained calcite-bearing sample increases in the first $1 \%$ strain then decreases by $\sim 40 \%$ between 1 and $5 \%$ strain, and after $5 \%$ strain stays the same. In the sample with fine-grained calcite, ice grain size decreases by $\sim 30 \%$ reaching $5 \%$ strain and then stays the same.

Graphite-bearing ice shows a different behaviour, with a steady $30 \%$ decrease in ice grain size in first $12 \%$ strain, and little changes thereafter (Fig. 12). Bulk grain size reduction is more pronounced in the pure ice sample, where the starting grain size is reduced by half (Fig. 12; Table 2). The final grain size of coarse-grained calcite-bearing ice is smaller than that of pure ice $(170 \pm 13$ and $272 \pm 30 \mu \mathrm{m}$, respectively), and samples with fine-grained calcite and graphite show even smaller final average grain sizes (147 \pm 14 and $146 \pm 17 \mu \mathrm{m}$, respectively; Fig. 12).

At a medium strain rate (Fig. 12), there are well-defined trends in grain size evolution of ice with different initial grain sizes. In the pure ice, 3-D experiments, the mean grain size $(\sim 270 \mu \mathrm{m})$ after $20 \%$ shortening (Table 2$)$ has stabilized. The development of this stabilized grain size is independent of initial variations in grain size and appears to be dependent of strain rate as also pointed out by Peternell and Wilson (2016). However, the rates of grain size change are perturbed by localized fluctuations, which can be accounted for by grain nucleation with grain growth. These fluctuations stabilize with the development of the bimodal grain size distributions observed in the final microstructure (Figs 10b, 11b). Initial ice grain sizes also do not control the final mean grain size in the two-phase samples. Instead the ice grain size is controlled by the size and distribution of particles. In the 2-D observations (FA in Fig. 12) of the constant and long-cycle strain rate deformation of pure ice produce comparable grain sizes $(\sim 320 \mu \mathrm{m})$ at the 
a) Bubble ice - fast (MD11)

Mean grain size $372 \pm 13.6 \mu \mathrm{m}$

Aspect ratio 1.39

$\mathrm{n}=185$

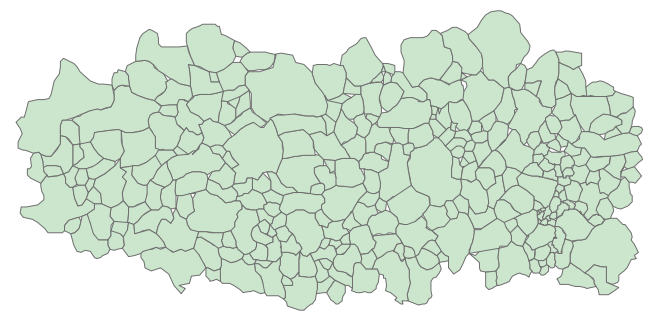

b) Pure ice - fast (MD4)

Mean grain size $178 \pm 4.1 \mu \mathrm{m}$

Aspect ratio 1.37

$n=170$

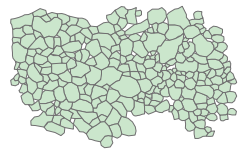

c) Pure ice - medium (MD10)

Mean grain size $246 \pm 9.3 \mu \mathrm{m}$

Aspect ratio 1.38

$\mathrm{n}=300$

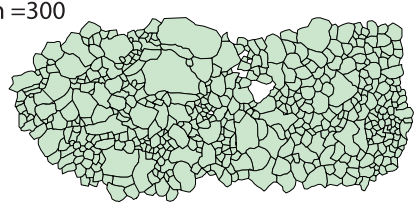

d) Calcite-ice - fast (CC_05)

Mean grain size $201 \pm 7.3 \mu \mathrm{m}$

Aspect ratio 1.43

$\mathrm{n}=245$

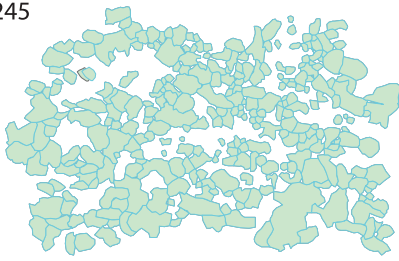

e) Graphite (20\%)-ice - fast (G_06)

Mean grain size $237 \pm 5.6 \mu \mathrm{m}$

Aspect ratio 2.04

$\mathrm{n}=218$

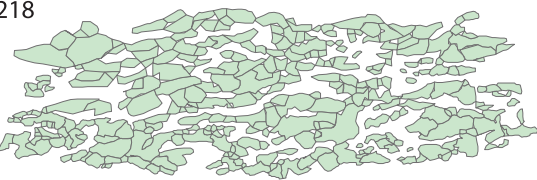

f) Graphite (40\%)-ice - fast (GG_03)

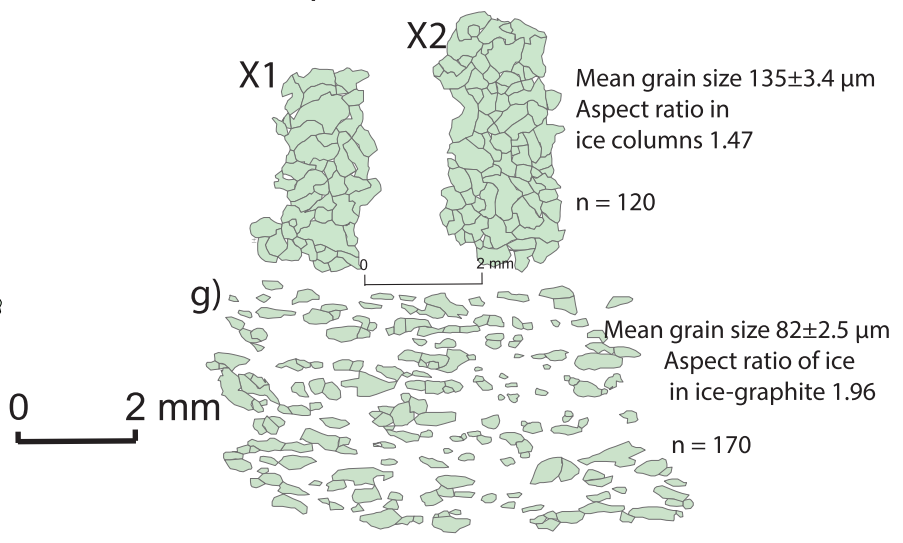

Fig. 10. Digitized images comparing grain sizes and aspect ratio for ice domains in bubble, pure ice and where there was a dispersed second phase. The background in (d) is fine calcite with grain size $<150 \mu \mathrm{m}$. The background in (e) and (g) is graphite. $n=$ number of grains digitized using ArcGIS from fabric analyser images. Bar scale of $2 \mathrm{~mm}$ applies to all images.

termination of an experiment. This is slightly larger than the 3 -D average grain size $(\sim 270 \mu \mathrm{m})$. In the short-cycle experiments, there is a reversal with the average grain size less $(\sim 215 \mu \mathrm{m})$ than the other pure ice experiments.

Grain size is not the only measurable property in the 3-D neutron deformation experiments, we can also extend our observations to quantify the nucleation of new grains (Fig. 6). The seeding rate for new grain nucleation progressively increases during deformation and stabilizes after $\sim 10 \%$ strain in both the pure ice and in the ice with dispersed second phases (Fig. 7). However, the general fluctuations in grain size (Fig. 11) and observation of bimodal grain distributions (e.g. Fig. 11b) is an indication that nucleation of new grains vs grain boundary migration has not reached steady state with ongoing deformation. Therefore, recrystallization is increasing with the progress of deformation, dislocations are removed until the rates of dislocation generation and removal are equal, and the seeding rate for grain nucleation is balanced as indicated by the constant nucleation rate of new grains (Figs $7 d, e, 8$ ).

\section{PREFERRED ORIENTATION DEVELOPMENT}

It is well known that the strength and position of the [c]-axis distributions in ice depend on the amount of strain (e.g. Wilson and Russell-Head, 1982; Piazolo and others, 2013;
Qi and others, 2017). To quantitatively assess the final [c]-axis fabric development at different strain rates in the 3-D experiments, data were evaluated from partial and full pole figures and compared with results obtained via optical means. The optical evaluation was from vertical sections ( 300 $\mu$ m-thick), cut parallel to the compression direction, after full textural analysis was undertaken. Individual [c]-axis orientations and quality values (Peternell and others, 2009) were analysed in axial distribution diagrams (AVA - 'Achsenverteilungsanalyse') using the software 'Investigator' (Wilson and others, 2007). The [c]-axis orientation measurement for each grain was evaluated in the AVA image, following the principles outlined in Wilson and others (2007) and Hammes and Peternell, (2016). Such an analysis was also applied to the continuous and synchronous recording of microstructure and crystal $[c]$-axis orientations recorded during the 2-D experiments.

As neutron diffraction derives the combined bulk CPO of all phases in the sample, their individual diffraction reflections must be resolved or separated through diffraction peak fitting analysis. For ice, the experimental data from (002), (100) and (101) reflections were plotted using MTEX texture analysis (Hielscher and Schaeben, 2008), and a $5^{\circ}$ Gaussian smoothing function, as $[c]$ and $\langle a>$-axis pole figures (Figs 2-5) using ODF reconstructions (e.g. Wenk, 2006). The (002) poles obtained from the graphite represent 

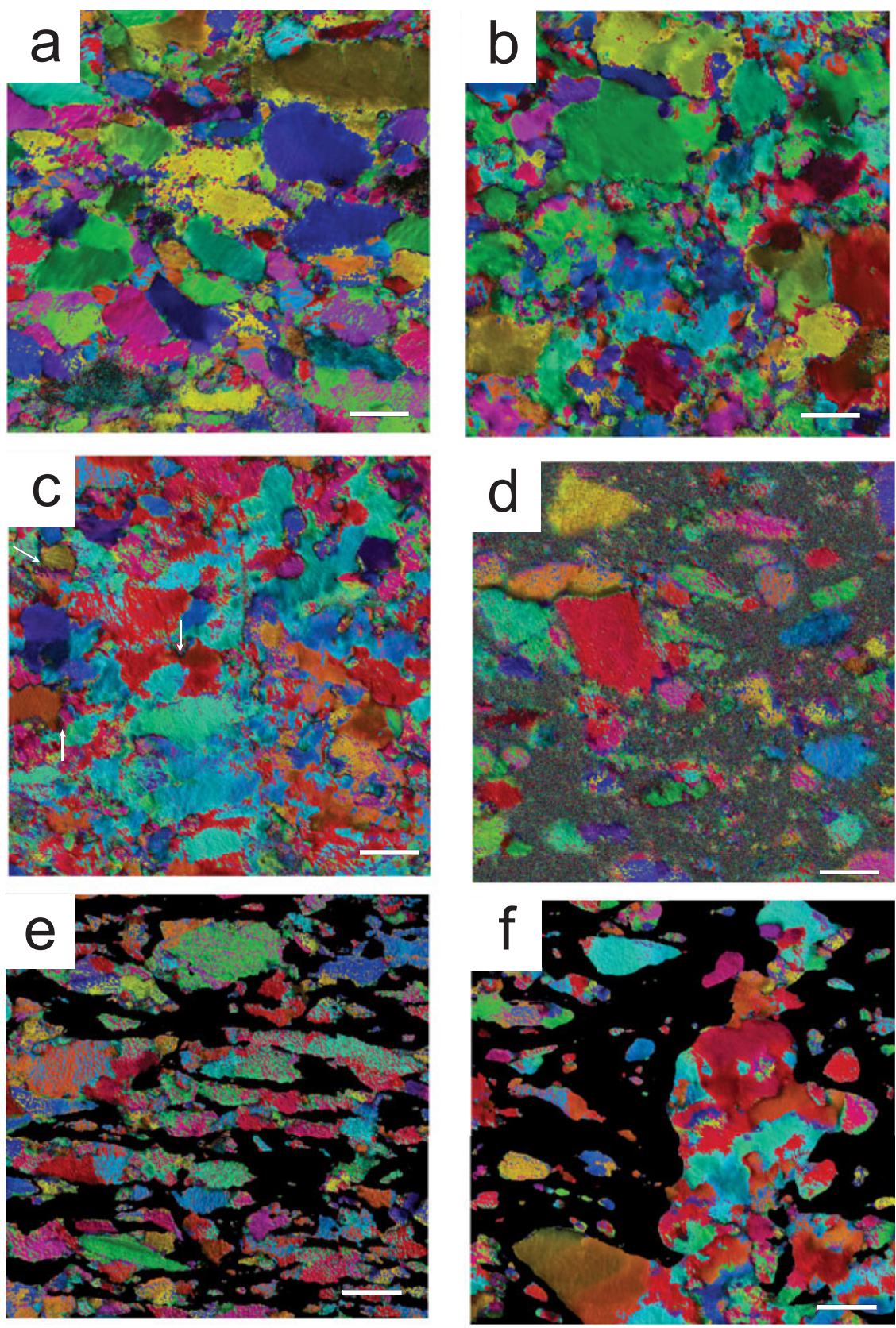

Fig. 11. Enlargements of key areas within representative deformed samples from orientation images described in the text. (a) Bimodal grain size distribution in bubble ice (Fig. 3b), with large tabular-shaped undulose grains and smaller recrystallized grains. (b) Lobate interlocking grains in bubble ice (Fig. 3d) displaying undulose extinction. (c) Ice with coarse calcite (Fig. 4b) dominated by irregular-shaped grains that can be related to pinning of grain boundaries by the calcite as indicated by the white arrows. (d) Isolated irregular ice grains displaying intracrystalline distortions within fine ice-calcite matrix (Fig. 4e). (e) Isolated ice within graphite matrix (Fig. 5a) exhibit undulose extinction and subgrain development. (f) Larger irregular recrystallized grains with undulose extinction (Fig. 5d) within the ice only channels in the graphite-ice mixtures (Fig. $5 \mathrm{~d}$ ). Bar scale for all micrographs is $0.1 \mathrm{~mm}$.

the [c]-axis distribution of the graphite flakes after deformation (Fig. 5). All pole figure density distributions of the deformed ice confirm an axial symmetry, with [c]-axis poles preferentially aligned with respect to the compression direction. However, there are significant differences between samples deformed under different strain rate conditions, which will be discussed below.

\subsection{Fabrics in bubble and pure ice samples}

The distribution of $[c]$-axes in the starting material for the pure (Fig. 2a) and bubble ice (Fig. 3a) is very close to random. By the end of these experiments (Figs 2, 3), the optically determined [c]-axis pattern (fabric analyser data in Fig. 3) was variably recognizable. When compared with the distinct small-circle girdle patterns around the compression axis $\left(\sigma_{1}\right)$ recognized in the bulk neutron diffraction results. In the pure ice fast experiment (Fig. 2b), the optically determined $[\mathrm{C}]$-axis pattern contains distinctly different geometries and intensities compared with the cones (small circles) parallel to the compression axis in the neutron diffraction data. This is attributed to the poor geometrical quality obtained by the fabric analyser, as the grains have strong intracrystalline distortion and no indication of grain 


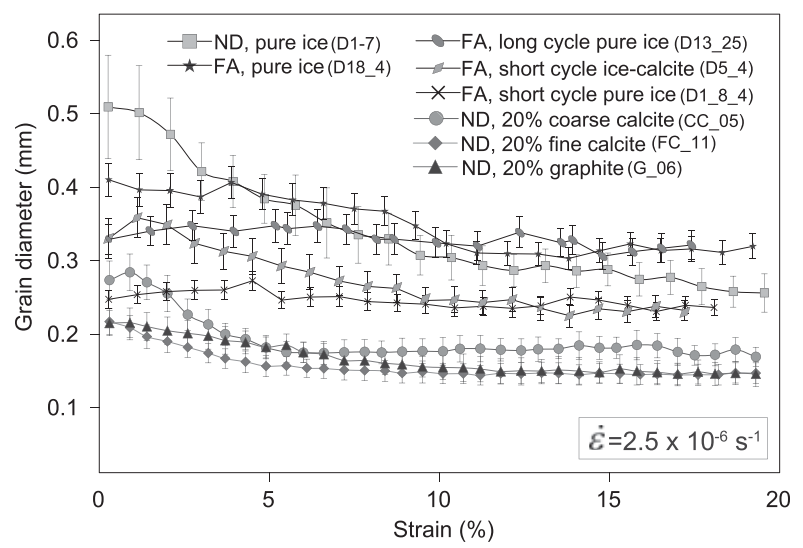

Fig. 12. The evolution of the average grain size of ice at small strain steps at strain rate $2.5 \times 10^{-6} \mathrm{~s}^{-1}$ (medium) obtained by neutron diffraction(ND) analysis and during in situ experiments on the fabric analyser (FA) and deformed at $-7 \mathrm{C}$. The average grain size is defined as a 3-D equivalent diameter. The error bars are between 15 and $20 \%$.

boundary migration (see Appendix). In the medium strain rate experiment (Fig. 2c), where there was notable grain boundary migration, there was a better correspondence between the optical and neutron diffraction data. The $<a>$-axes are distributed in a broad swath along the plane perpendicular to the compression axis.

In the bubble ice experiments (Figs 3a-d), the [c]-axis distributions are markedly different to the pure ice with lower J-index values. In the fast experiment, where sample was shortened $11 \%$ (Fig. 3b), the CPO consists of a strong concentration parallel to the compression axis. The optically derived results are comparable to the neutron diffraction data, with a small number of large tabular grains related to the strong maxima parallel to the compression axis. The [c]-axis of the smaller grains differ from those of the larger grains in that the $[c]$-axis positions are more dispersed in a wide zone across the entire girdle that surrounds the compression axis (Fig. 2b). In contrast, a comparable medium experiment (Fig. 3c) produced a stronger CPO with [c]-axes preferentially clustered or at a small angle around the compression axis. The corresponding $\langle a\rangle$-axes are concentrated in the broad swath girdle perpendicular to the compression axis. With $\langle a\rangle$-axes in the medium strain rate experiment having distinct sub-maxima at 45 and $90^{\circ}$ to the compression axis. In a higher strain $(\varepsilon=36 \%$ ) fast experiment (Fig. $3 d$ ), the J-index, which is a measure of the degree of preferred orientation or 'sharpness' in the ODF (Sturcken and Croach, 1963), marginally increases with initiation of two concentrations of $[c]$-axes symmetric and a low angle to the compression axis.

In the medium strain rate 2-D fabric analyser experiments (Fig. 9a) and long-interval strain rates cycle experiments (Fig. 8b), the CPO forms an intermediate girdle-cluster pattern symmetrically related to the compression axis. The short-interval strain rate cycle experiments (Figs 9c, d) show a fine grained, bimodal grain size distribution, with crystal orientations almost unchanged and close to random.

\subsection{Fabrics in ice-calcite samples}

All deformed ice-calcite samples analysed by neutron diffraction have non-random CPOs with $[c]$-axes concentrated in cones about the compression axis (Fig. 4). The fast experiments (Figs 4b, e) exhibit [c]-axis maxima within the cones, which are aligned $\sim 45^{\circ}$ to the compression axis. In contrast, with a medium strain rate and 20 vol.\% calcite (Figs 4c, d), most grains are strongly aligned in a cone about the compression axis with no sub-maxima. However, where there is 40 vol.\% calcite, this cone-like distribution and J-index is weaker. Elements of these patterns are seen in the optical data obtained from the fabric analyser.

The $<a>$-axes lie in a broad girdle perpendicular to the compression axis $\left(\sigma_{1}\right)$ with discrete concentrations only in the fast experiment (Fig. 4e). These concentrations reflect the four dominant $[c]$-axis distributions about the compression axis. With increasing fine calcite (40 vol.\%), the [c]axis distributions and the J-index are weaker.

In order to investigate the character of CPO development with progressive strain, we have used fluctuations in [002] intensities. Figure 7 shows the large variability of intensities with progressive deformation from a random initial $[c]$-axis preferred orientation (at $0.25 \%$ shortening). At a finite strain of $6.7 \%$ shortening, the intensity then decreases at $16.8 \%$ shortening. This decrease suggests the nucleation of new recrystallized grains, which progressively grow to produce the increased intensity by the end of deformation at $20.5 \%$ shortening. The final [c]-axis pattern of the ice crystals is a strong small-circle concentration about the deformation axis (Fig. 4).

\subsection{Fabrics in ice-graphite samples}

Non-random CPOs with ice [c]-axes concentrated in cones about the compression axis (Fig. 5) are a feature in all icegraphite samples. There is a reasonable degree of correspondence between neutron diffraction and fabric analysis data for samples with $20 \%$ graphite, but not with the fabric analysis measurements in the $40 \%$ graphite. In a medium strain rate experiment stopped at $6 \%$ strain (Fig. 5c), there is a noticeable alignment of $[c]$-axes at $\sim 45^{\circ}$ to the compression axis. Shortenings of $20 \%$ during a medium deformation, with 20 vol.\% graphite (Fig. 5b), produce higher J-indices than the corresponding fast experiment (Fig. 5a) or where there is 40 vol.\% graphite (Fig. $5 d$ ).

Graphite fabric intensities, expressed as multiples of random orientation, reach 6.5 at strains of $6 \%$ shortening (Fig. 5c) and increase to 11 during the medium strain rate experiments (Fig. 5b). These higher intensity values also reflect higher degrees of preferred orientation and as seen in the J-index. With increasing strain graphite CPOs in samples with 40 vol. $\%$ graphite, the radius of cone angles decreases from $55^{\circ}$ (Fig. 5c) to $30^{\circ}$ (Fig. $5 \mathrm{~d}$ ). Comparing fast (Fig. 5a) vs medium (Fig. 5b) strain rates also reflect increasing higher degrees of preferred orientation. The effect of increasing graphite content from $20 \mathrm{vol} \%$ (Figs $5 \mathrm{a}, \mathrm{b}$ ) to $40 \mathrm{vol} . \%$ (Figs $5 c$, d) makes little difference in the radius of the cone angle $\left(42^{\circ}\right)$; however, the graphite intensity decreases from 11 to $\sim 8$ reflecting a lower degree of preferred orientation.

\subsection{The evolution of the $[c]$-axis $\mathrm{CPO}$}

CPO neutron diffraction data are presented in terms of $X Y$ intensity plots (Fig. 13a) of orientations at a given angle $(\chi)$ to $\sigma_{1}$ along cross-sections in complete (Fig. 13) and incomplete (0001) pole figures (Figs 13a-d). The absence of significant CPO in the undeformed pure ice (D5_2, Fig. 13a) is 

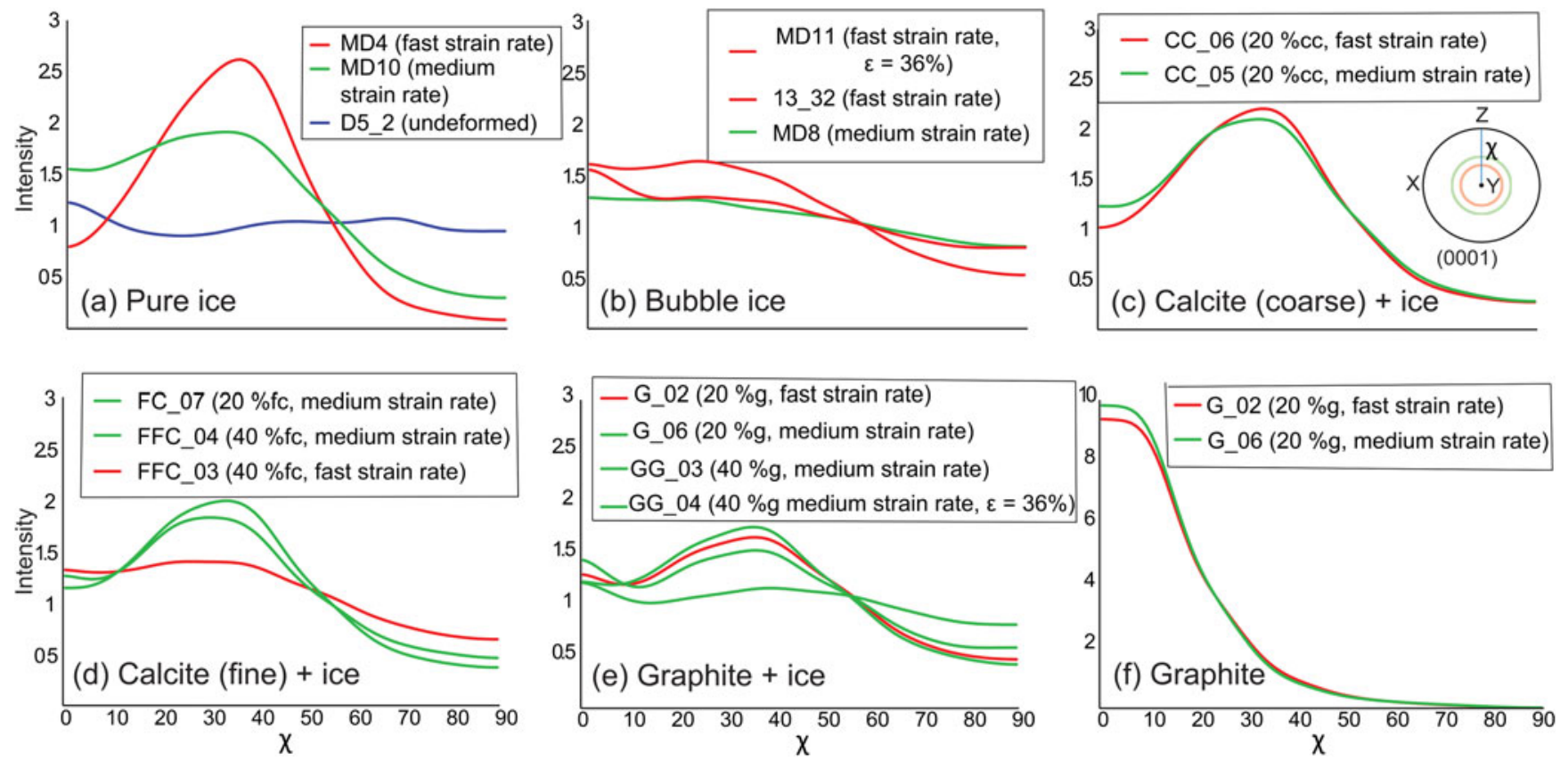

Fig. 13. Summary of crystal orientation data from neutron diffraction uniaxial compression experiments. Inset in (c) shows results in a CPO with circular symmetry around the $X$-direction of the pole figure, the area of measurement for final pole figures and representative crosssection (N-S line), $\chi$ - vertical azimuth of $[c]$-axis orientation in respect to $\sigma_{1}$. (a-f) Cross-sections of pole figures showing final CPO of (0001); the strength of the CPO is defined by J-index of the ODFs that are calculated with half-width and resolution of $5^{\circ}$. All crosssections are calculated after 20\% shortening except for MD11 ( $\varepsilon=36 \%)$ and GG_04 $(\varepsilon=6 \%)$. cc, fc and g represent vol.\% of coarse calcite, fine calcite and graphite, respectively.

confirmed by the very low-intensity troughs at $\chi \sim 25^{\circ}$ and uniform intensities across the higher angles in the crosssection. Fast deformation of pure ice (MD4) to 20\% shortening develops a well-defined high-intensity peak at $\chi \sim 35^{\circ}$ (Fig. 13a), which is higher than the comparable medium strain rate experiment (MD10). This peak is accompanied by low intensities at low and high $\chi$ angles. The bubble ice (Fig. 13b), deformed to $20 \%$, at both fast (13_32B) and medium (MD8) strain rates, displays similar flat intensities that decrease towards high $\chi$ angles. It is only after $36 \%$ shortening (MD11) that the CPO intensity starts to increase.

In the calcite-ice samples, the intensity (Figs 13c, d) of the peak of (0001) at $\chi \sim 35^{\circ}$ is lower than in pure ice, and the number of orientations at $\chi \sim 80^{\circ}$ is higher. In ice with coarser calcite deformed to $20 \%$ (Fig. 13c), the CPO develops a well-defined high-intensity peak at $\chi \sim 35^{\circ}$ under both fast (CC_06) and medium (CC_05) strain rates. This is similar to fine ice deformed at the medium strain rate (FC_07, Fig. 13d). In contrast, the intensity of the ice with a finer calcite powder is distinctly lower and broader when the calcite content is increased to 40 vol.\% (FFC_03, FFC_04; Fig. 13d).

In the graphite-ice samples (Fig. 13e), with 20 vol.\% graphite as second phase, it is the medium strain rate (G_06) that produces a slightly higher intensity at $\chi \sim 40^{\circ}$ than the fast (G_02) deformation. These are well-defined peaks in comparison to the lower intensities and off-set peaks observed with only $6 \%$ strain $\left(\mathrm{G}_{-} 04\right)$. Because the graphite is reoriented due to its tabular grain shape, this may explain why the intensity peak is offset $\left(\chi=\sim 5^{\circ}\right)$ from that of pure ice. The (001) pole figures for graphite after deformation (Fig. 13f) are all characterized by a high-intensity axial symmetric distribution in the direction of $\sigma_{1}$.
The evolution of the CPO development in the 3-D experiments can be derived from measurements taken from partial pole figures (Figs 14a-d). The partial pole figure is measured from $\chi=0^{\circ}$ to $\chi=40^{\circ}$, at selected time intervals (Table 2) and used to obtain the neutron diffraction grain size (Fig. 11) and textural evolution (Figs 14a-d). As the maximum intensity of (0001) orientations lies at $\chi<40^{\circ}$, we are able to characterize the developing CPO. The incomplete pole figures show the increasing intensity of the (0001) maximum at $\chi \sim 30-35^{\circ}$, with progressive deformation. In the ice-only sample (Fig. 14a) and sample with coarsegrained calcite (Fig. 14b), this peak becomes visible at $\sim 5 \%$ shortening, whereas in ice with fine-grained calcite (Fig. 14c) and graphite (Fig. 14d) the peak forms at strains of $\sim 10$ and $15 \%$, respectively. In the 2-D experiments, $\mathrm{CPO}$ development was derived using the eigenvectors of the orientation tensor (Scheidegger, 1965; Woodcock, 1977) at each increment of strain (Peternell and Wilson, 2016) and plotted on a ternary PGR diagram (Point-GirdleRandom diagram, Vollmer, 1990; Fig. 14e). In the constant strain rate experiments on pure ice, the E1 eigenvector vector shifts from a random orientation towards a girdle after $>4 \%$ shortening (Fig. 14e). Where there are short-interval changes in strain rate (Fig. 14f) and/or the presence of calcite (Fig. 14g), there are only minor changes in the nature of the CPO.

The J-index correlates well with the above observed changes, in other words the overall intensity and sharpness of the CPO peak is strongest for pure ice and weakest for the bubble ice and graphite-bearing ice (Fig. 15). Fast strain rates are the major contributor to the $\mathrm{CPO}$ strength in both the pure and bubble ices. In contrast, medium strain rates contribute to the higher J-index values observed in ice with 


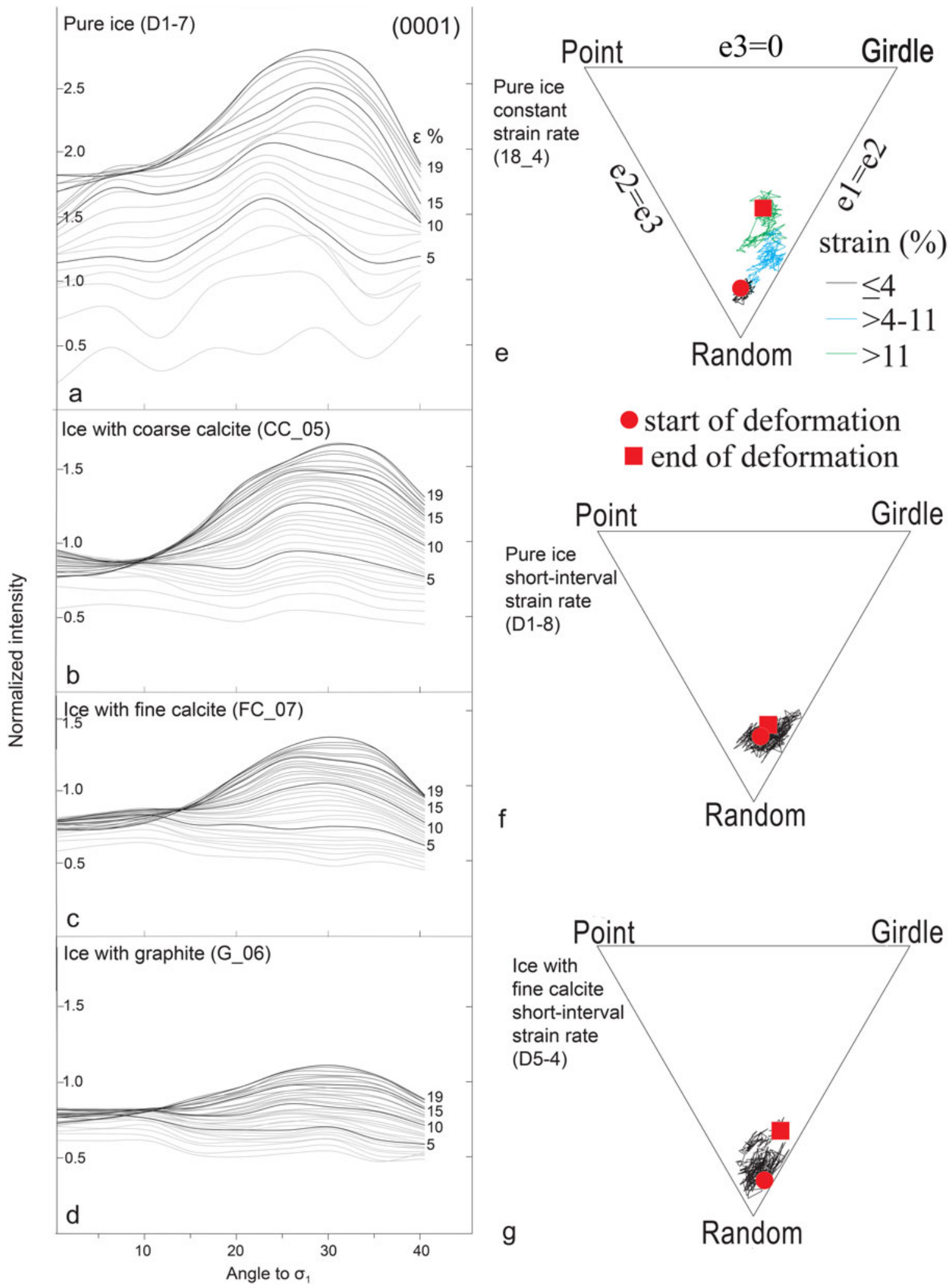

Fig. 14. Partial pole figures of (0001) obtained during the 3-D and PGR (point, girdle, random) ternary diagrams (Vollmer, 1990) from 2-D experiments illustrating the evolution of the (0001) CPO with increasing strain. (a-d) Neutron diffraction data as a function of strain and second-phase composition (modified after Cyprych and others, 2016). (e-g) PGR diagrams, based on [c]-axis pole figures in (Fig. 9) and calculated from the normalized eigenvalues of the orientation tensor (Vollmer, 1990), and estimating the degree to which [C]-axes define a point $(P)$, girdle $(G)$ or random $(R)$ distribution. A characteristic 'CPO path' defining the systematic change of CPO from start to termination of deformation at $\sim 20 \%$ shortening.

a dispersed second phase. Dispersion of 20 vol. \% fine calcite or graphite in ice has a greater effect than coarser calcite. As the volume proportion of second phase is increased to $40 \%$, this obviously restricts CPO development. This is more obvious where there are interconnected layers of a weak material such as graphite (Fig. 15). 

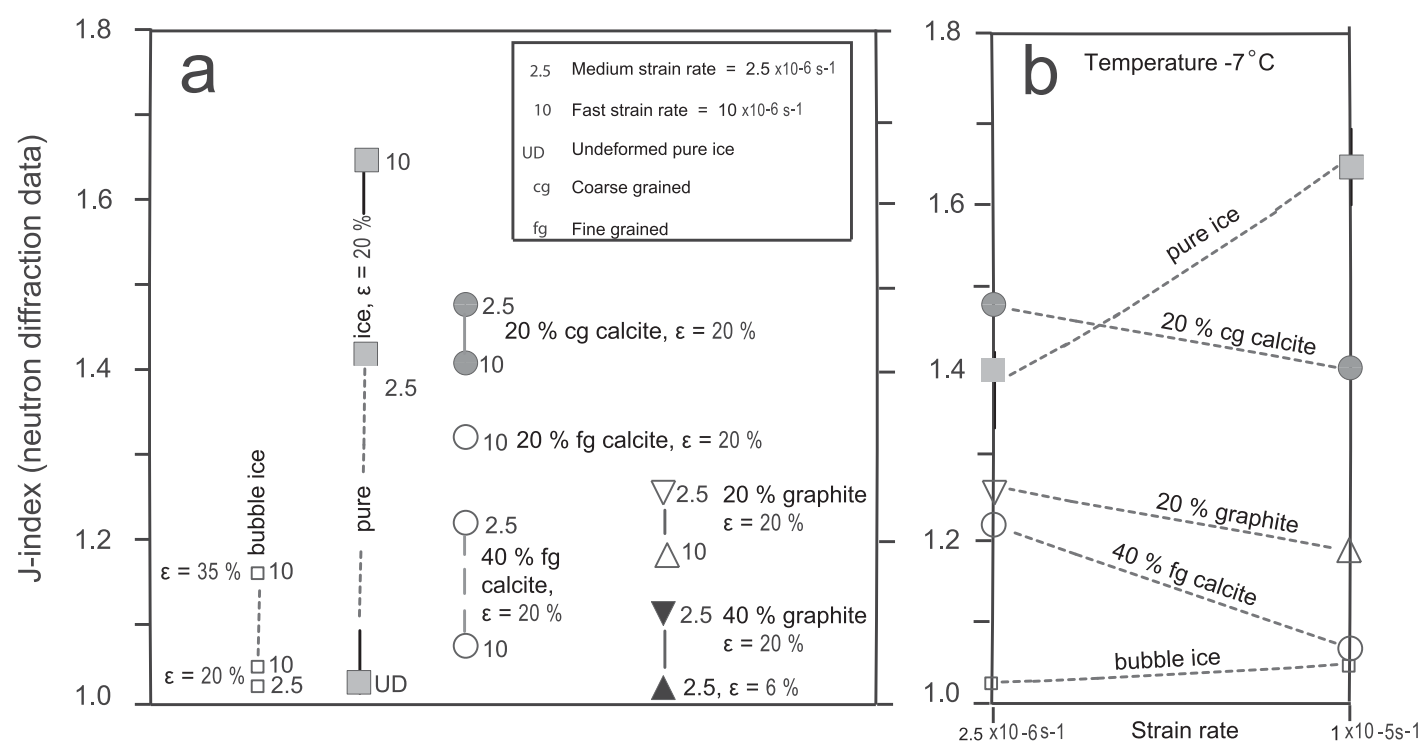

Fig. 15. Plot of J-index variation for ice from neutron diffraction data as a function of strain rate and second-phase composition. (a) Comparative plot of data for strains $(\varepsilon)$ of $20 \%$ except for one sample of bubble ice (MD11; $\varepsilon=36 \%$ ) and a sample containing 40 vol.\% graphite $\left(G G \_04 ; 6 \%\right)$. (b) Plot of J-index intensity vs strain rate for samples deformed $20 \%$. Strain rate sensitivity is seen in the pure ice where a fast strain rare has the highest J-index. A decrease in strain rate produces the highest intensities where there is a second phase.

\section{DISCUSSION}

\subsection{Deformation and recrystallization mechanisms in the $\mathrm{D}_{2} \mathrm{O}$ ice}

The microstructure of the pure ice at a fast strain rate shows evidence for operation of dislocation creep processes and the production of CPOs with the highest J-index (Fig. 15). The results in the pure ice are also representative of what is happening in the matrix of samples containing dispersed calcite and graphite. There is a widespread formation of optically visible subgrain substructures, wavy extinction and grain boundary migration in all investigated samples (Fig. 11). Analyses of these microstructures by EBSD (Cyprych and others, 2016) indicates that most of the strain is produced by dislocation creep occurring as a glide on the easy basal $<a>$ slip system.

In the medium strain rate experiments, grain boundary migration has been observed to accommodate basal $<a>$ slip, but produces a weaker CPO (Figs 13, 15). Grain boundary migration is particularly obvious when deformation proceeds beyond the peak stress. This involves processes competing between grain size increase due to surface energy reduction, internal energy reduction and grain size reduction due to strain-induced nucleation. During a mechanical steady state, grain growth and grain size reduction processes balance, involving nucleation of new grains, and yields a constant flow stress (Figs $7 d, e, 8)$. We believe that the grain size evolution is not steady state (Fig. 7), but represents an equilibrium between grain size reduction and grain growth processes, involving subgrain boundary rotation and grain boundary migration. There may also be the influence of either lattice strain energy or surface energy (e.g. Urai and others, 1986; Derby and Ashby, 1987; De Bresser and others, 1998) in the deformed material. However, we do not recognize the temperature-dependent static grain growth described by Alley and others (1986).

In the fast deformation of the bubble ice, during the first $10 \%$ of shortening, a different microstructural evolution (Fig. 2b) occurs before reaching a peak stress. There is a lack of strong intracrystalline distortions and pronounced clustering of $[c]$-axes parallel to the compression axis $\left(\sigma_{1}\right)$, which may reflect the role of the pore spaces attributed to the bubbles. These pores probably accommodated external rotation and the formation of elongate grains, exhibiting wavy grain boundaries (Fig. 2b), with a dominant activity of $[c]$ as a slip direction, before the onset of normal crystal plastic mechanisms. Initially some processes, such as surface or volume diffusion, would rapidly accommodate the pores or crystal overlaps. This strong single maxima CPO development seen in the bubble ice is remarkably similar to what has been observed in the initial stages of deformation of pure ice, where there is also removal of voids. From partial pole figure data obtained in the first $1.5 \%$ shortening in the pure ice, there is a consistent weak but dominant component of $[c]$-axes parallel to the compression axis (e.g. Piazolo and others, 2013).

The microstructures in the bubble ice, and the accompanying strong single maxima CPO (Figs 2b, c), are comparable to features developed in 'polar firn' (Kipfstuhl and others, 2009) or from dissolution-precipitation creep in quartz (den Brok, 1996). This raises the question of what driving forces dominate during the initial stages of deformation in the bubble ice. Is it possible that differences in elastic deformation (stress) between the grains in the bubble ice are controlled by dissolution or growth rate (cf. Kamb, 1959) similar to processes of precipitation and dissolution? Den Brok (1996) pointed out that if stress is important then it will be grains with a small angle between [c]-axis and the Z-axis of shortening that exhibit the least amount of dissolution, whereas grains $\sim 50^{\circ}$, to $Z$ manifest the highest degree of dissolution. An alternative model has been proposed by Kipfstuhl and others (2009) for 'firn' that dynamic recrystallization is achieved by strain-induced boundary migration, where the largest grains (e.g. Fig. 3b) steadily increase their cross-sectional area by rapid migration of old grain boundaries through a build-up of dislocation densities. However, as pointed out by Brumberg and others (2017) at the solid-vapour interface of a bubble, surface energies are 
the fundamental connection between macroscopic shape and molecular configuration. Such a connection in ice is dominated primarily by prism and basal faces (Brumberg and others, 2017) with mechanical stresses strongly impacting the shape (Handel and others, 2008). This may explain the initial shape and CPO structure in the bubble ice.

A marked change in microstructure occurs in the bubble ice deformed to higher strains (e.g. $\varepsilon=36 \%$; Fig. 3d) with the nucleation of new grains. This appears to be driven by lattice strain energy and grain boundary surface energy. The grains are interlocking, substructures are abundant, and grain boundaries have irregular shapes, without any memory to its former structure and developing a CPO comparable to that of the pure ice. It is important to appreciate that at $T=-7^{\circ} \mathrm{C}$ the generation/build-up of dislocations by dislocation climb and glide and elimination of dislocations by new low dislocation nuclei and their subsequent growth are competing as mechanisms, but the relative contribution from each changes as external conditions change. As pointed out by Piazolo and others (2013), more than one mechanism or process will dominate at different stages in the rheology (Fig. 7d) and these are dependent on the strain rate and can be identified in intensity profiles (Figs $7 \mathrm{a}-\mathrm{c})$. In Stage 1, there is a grain boundary migration with relatively little change in grain size. During Stage 2 , there is a general grain size reduction. Stage 3 is characterized by a decrease in the rate of grain size reduction. During Stage 4, grain size shows a general stabilization with some fluctuations and slight grain size increases as there is a competition between grain nucleation and grain growth.

\subsection{Microstructural differences in relationship to second-phase content}

Even though the overall behaviour of the ice involves the onset of intracrystalline plasticity, involving dislocation glide, the rheology (Fig. 8) is markedly sensitive to composition and volume fraction of a second phase. Compared with pure ice, with volume fraction of $>20$ vol. $\%$ or change from coarse to fine calcite, there was mechanical hardening (cf. Table 1). This has been attributed to viscous drag in the ice as it flowed around particles (Durham and others, 1992). In such a situation, impurities can become localized at grain boundaries (e.g. Fig. 11c) and impede boundary movement if driving forces are not sufficient to allow the boundaries to move past them. These dispersed phases produce localized rheological instabilities that commences with dislocation creep but deformation is rapidly accommodated by diffusion creep.

Due to the pinning effects of secondary particles, grain growth is effectively prohibited and the matrix grain size is kept relatively fine (e.g. Figs $10 \mathrm{~d}, 11 \mathrm{~d}$ ). Pinning by secondary particles also suppresses the flow and makes the ice stiffer. For instance, the ice-graphite samples initially exhibited strain hardening, but the stress-strain curve stabilized (Figs $8 \mathrm{~b}, \mathrm{c})$ and there was a little weakening in comparison to the pure ice. In ice-calcite samples deformed at finite strains $>20 \%$ shortening, there is also further hardening (Fig. 7e). The application of in situ textural analysis (Figs 7a-c) also allows one to correlate $\mathrm{CPO}$ development with progressive strain and microstructural processes. Fluctuations in textural intensities and character from a random initial [c]-axis preferred orientation (at $0.25 \%$ shortening) occur with progressive deformation. At a finite strain of $6.7 \%$ shortening, intensity increased then decreased at $16.8 \%$ shortening. This decrease suggests nucleation of new recrystallized grains, which progressively grow to produce an increased intensity by $20.5 \%$ shortening. Even though the stress-strain relationship is steady state after $\sim 10 \%$ strain, the presence of calcite extends the process of new grain nucleation (stage 3; Fig 7e) in comparison to pure $\mathrm{D}_{2} \mathrm{O}$ (Fig. 7d).

Our study and that of Peternell and Wilson (2016) indicate that pinning by second-phase inclusions does significantly restrict grain boundary migration and CPO development during deformation. This is supported by observations in many ice core studies (e.g. Gow and Williamson, 1976; Gow and others, 1997; Durand and others, 2006). However, these observations are contrary to the microstructural conclusions reached by Eichler and others (2017) in a study of four polar ice cores. They suggested that there is no significant correlation between grain boundaries and inclusion distributions. Unfortunately, Eichler and others (2017) fail to consider the difficulties of preserving deformation microstructures in ice cores because of stress and temperature differences post-deformation. It is probably for this reason that natural ice grains overgrow inclusions, by a process of static annealing as described by Alley and others (1986).

Measurements of the changes in shapes of the bubble ice and pure ice are approximately the same (aspect ratios 1.38; Figs $10 \mathrm{a}-\mathrm{c})$. However, there is a notable increase in grain boundary migration and the production of larger grains during the slower deformation experiments (Fig. 10c). Increased second-phase content inhibits effective grain growth by pinning of grain boundaries and this leads to weaker ice CPOs with original distributions of calcite producing a dispersed distribution of single ice grains (Fig. 10d).

Ice with soft particulates, such as graphite (Fig. 5), has microstructures and localized CPOs that depend on the distribution of the harder ice phase. Exclusive ice regions ( $X 1$ and $X 2$ in Fig. 10f) are recrystallized and their boundaries indicate extensive grain boundary migration (Fig. 11f). In the graphite-ice matrix, the ice occurs as either elongate aggregates (Figs 10e, 11e) or as dispersed isolated grains with undulose extinction with aspect ratios of $\sim 2$. Interparticle distance between the ice grains increases with 40 vol.\% graphite with a marked grain size reduction and grain shape variation (Fig. 10g). The graphite pins the ice grain boundaries and there are variations in grain shape as a result of increasing contact with graphite grains. As the abundance of graphite decreases, the rate of grain boundary migration and localization increases (Figs 10e, g) and the CPO increases (Fig. 5b). The weak CPOs in the 40 vol.\% sample can be explained by Zener drag forces (Herwegh and Berger, 2004) from the second phase and lack of strain accommodation in the ice grains may suggest that strain was more easily accommodated in the adjacent graphite.

\subsection{CPO development in relationship to strain rate conditions}

Considerations of combined strain rate and different dispersed phases in ice have several implications, first for the interpretation of CPOs and second for the rheology in natural ice masses and rocks. The behaviour of the twophase materials at a constant temperature is strongly dependent on strain rate, mineral volume proportion and stiffness of the second phase. In a monomineralic aggregate (e.g. pure or 
bubble ice), the greatest degree of CPO development (Fig. 15) occurs during the fast strain rate regime. In contrast, after a second phase is added, the highest CPO intensities are recorded in the slower strain rate experiments (Fig. 15). These $\mathrm{CPO}$ intensities diminish with reduction in grain size of the second-phase particles (e.g. coarse vs fine calcite; Fig. 14b vs Fig. 14c) or with an increase in volume fraction of second phase (20 vs 40 vol.\%). At a constant strain rate, this probably reflects a switch from fully viscous and fast flow in the mono-mineralic material, facilitated by dislocation glide plus climb, to the onset of slower processes accommodated by diffusion and/or by the nucleation and growth of new grains, which may be hampered by the second phase. If there is a short-term cycling of strain rate, then identifiable CPO pattern development, in both pure ice (Fig. 9c) and calcite-rich ice (Fig. 9d), is impeded by the rate of grain boundary migration.

The 3-D samples with fine calcite exhibit a distinct strain hardening even at low strains (Figs 8b, c). This is also reflected in lower rates of CPO evolution (Fig. 14c) and CPO intensities particularly at fast strain rates (Fig. 13d). The accompanying grain size evolution in the 3-D sample (FC_11, Fig. 12) is also less than average grain sizes from the 2-D short-interval changes of strain rate (D5_4, Fig. 12), where CPO patterns are comparable with the initial random distribution (Figs $14 \mathrm{f}, \mathrm{g}$ ). The results from graphiteice samples reflect a less pronounced CPO development (Figs 13e, 14d) and a distinct rheological hardening at higher strains (Fig. 12), particularly if the graphite content is increased (Fig. 13e).

The CPO development is directly related to cumulative strain, crystal slip, disappearance of unfavourable orientations and sum of strain, strain rate, stress, bulk or rigid body rotation. The degree to which individual [c]-axes rotate is also governed by the distribution and nature of the particulate. With deformation, each ice crystal is initially accommodated by dislocation glide by basal slip plus climb and grain boundary migration. In the presence of a rigid particulate, the slip systems in individual grain can only accommodate a limited amount of rotation out of easy glide orientations before being consumed by neighbouring grains during grain boundary migration. In fact, grain boundary migration plays a central role in maintaining strain compatibility. Grains with high dislocation densities do not survive if grain boundaries can migrate. Whereas, if grain boundaries do not migrate and they survive, then the $\mathrm{CPO}$ is weaker.

\subsection{Strain partitioning processes in the ice}

The proportion and grain size of calcite and graphite particles have a pronounced effect on the deformation-induced grain size (Fig. 12), CPO evolution (Figs 13, 14a-d) as well as localizing strain in the ice matrix. It is the dissimilar rheologies of the constituent phases that gave rise to stress differences and strain incompatibilities within the ice aggregate. The bulk stress identified after each strain increment (Fig. 8) in these materials reflects the effects of domainal structural heterogeneities (Figs 10e, g) on stress and strain portioning within the aggregate. It is the second phases that hinder grain boundary migration by dragging or pinning the grain boundaries in the ice matrix (Herwegh and Berger, 2004). This then results in the stabilization of specific energetically favourable orientations and will enhance grain elongation parallel to the shape- preferred orientation of platy second phases (e.g. graphite, Figs $10 \mathrm{e}, \mathrm{g})$ to produce a foliation.

In the ice-calcite samples, there is a load-bearing framework of stronger calcite, particularly where there is a greater volume of fine particles, which produces isolated pockets in the weak ice phase (Fig. 10d). The stress dependence on strain rate in this strong phase significantly exceeds that in pure ice, so the bulk strain rate is concentrated into the weaker ice. The coalescence into pockets of fine calcite is enhanced by dynamic recrystallization in the weaker ice phase where stress concentrations become sufficiently large to induce unstable flow and elongation of the ice in the plane of flattening.

In the graphite-bearing ice, there are interconnected layers of weaker platy graphite grains deforming faster than the stronger ice phase (Figs 10e, g) and this is reflected in the higher stress exponent (Cyprych and others, 2016) than observed in the ice-calcite samples. Graphite deforms easily via basal slip, even at low temperatures and stresses (Soule and Nezbeda, 1968) and rotates to orientations perpendicular to $\sigma_{1}$ while the plastic strain accommodated by the ice is reduced. In the graphite-bearing samples, it also appears that strain compatibility is predominantly along incoherent phase boundaries and this leads to a higher stress in the ice and low nucleation rates in the stronger ice phase. Anisotropic grain boundary migration results in a high shape-preferred orientation of ice grains (Fig. 10e). As a result, a strong foliation and CPO (Fig. 5) defined by graphite develop. Local deviations from homogeneous strain occur in the immediate vicinity of inherited aggregates of ice $(X$ in Fig. 5a) or graphite ( $Y$ in Fig. 5c), probably reflecting localized differential stresses that are relaxed as the phases deform with respect to each other.

\section{CONCLUSION}

Our results indicate that, at a given temperature, the grain size distribution and the CPO development, as indicated by the Jindex, are sensitive to strain rate, via a competition between grain growth and grain size reduction processes in a dislocation creep regime. The flow of ice with particulates may be much more heterogeneous than generally assumed, as neither the $\mathrm{CPO}$ nor the optical microstructure clearly reveals the cause of the sensitivity to strain rate. This is only revealed when $\mathrm{CPO}$ intensities and rheologies are compared, in pure vs ice-carrying particulates, deformed under equivalent strain rates. Fast strain rates are the major contributor to $\mathrm{CPO}$ intensity and the weakening in pure ice, with weaker $\mathrm{CPO}$ intensities produced in pure ice under slower deformation conditions. In contrast, lower strain rates in materials with a second phase produce higher CPO intensities. These $\mathrm{CPO}$ intensities are reduced, while rheological strength of an aggregate increases, as the vol.\% of the second phase is increased or if there is a cycling of strain rate.

The presence of volume fractions with $>20 \%$ particulate phase is accompanied by a strength increase relative to that of pure ice. Inclusion of finer grained phases causes the ice to become more creep resistant with higher stresses necessary to achieve the onset of strain weakening. The strain-hardening behaviour described here is reduced as the applied strain rate is reduced. With relatively slower strain rates, there are more bimodal grain size distributions only developed in the final grain microstructure of the pure ice. Where there are finer particulates and platy phases, 
such as graphite, there is a greater restriction on grain growth and a greater development of deformation features such as subgrains within the enclosing ice. This may have implications for the prediction of the rheology of natural ice, which may be either weakened, in the case of bubble ice or firn, or strengthened by the presence of second phases.

Therefore, once constant physical conditions are identified, it is straight forward to obtain insights into the flow conditions of ice by evaluating the nature of the J-index. Lower values in a system containing a dispersed phase are thus ideal natural strain rate gauges, in combination with ice radar or seismic reflection data, for the identification of palaeo-deformation conditions in an ice sheet. We have further provided indications of transient conditions between diffusion processes during compaction of 'firn' (bubble ice) and the dislocation creep processes that operate in pure ice. Furthermore, the concepts presented here could provide new insights into the development of layering developed in glaciers and ice sheets that contain high particulate concentrations.

\section{ACKNOWLEDGEMENTS}

This work was supported by the Australian Nuclear Science and Technology Organisation through projects 1702, 2178 and 2910 and undertaken at Lucas Heights, Australia. The technical support and discussions with ANSTO staff and Daria Cyprych are gratefully acknowledged. SP acknowledges support from the Australian Research Council Future Fellowship (Project FT1100070). CJLW, MP and NH acknowledge support from the bilateral DAAD-AustraliaGermany Joint Research Co-operation Scheme (Project 57316937). Bill Durham and an anonymous referee made valuable comments that resulted in improvements in the paper.

\section{REFERENCES}

Alley RB (1992) Flow-law hypotheses for ice-sheet modelling. J. Glaciol., 38(129), 245-256

Alley RB, Perepezko JH and Bentley CR (1986) Grain growth in polar ice: I. Theory. J. Glaciol., 32(112), 415-424

Azuma N (1995) A flow law for anisotropic polycrystalline ice under uniaxial compressive deformation. Cold Reg. Sci. Technol., 23, 137-147.

Baker RW and Gerberich WW (1979) The effect of crystal size and dispersed-solid inclusions on the activation energy for creep of ice. J. Glaciol., 24(90), 179-194

Bindschadler RA and 27 others (2013) Ice-sheet model sensitivities to environmental forcing and their use in projecting future sea level (the SeaRISE project). J. Glaciol., 59(214), 195-224

Brumberg A and 8 others (2017) Single-crystal Ih ice surfaces unveil connection between macroscopic and molecular structure. Proc. Natl. Acad. Sci. 114(21) (doi: 10.1073/pnas.1703056114)

Bunge HJ (1982) Texture analysis in materials science: mathematical methods. Butterworths, London, UK

Castelnau O and 7 others (1998) Anisotropic behaviour of GRIP ices and flow in Central Greenland. Earth Planet. Sci. Lett., 154, 307-322

Cyprych D, Piazolo S, Wilson CJL and Luzin V (2016) Rheology, microstructure and crystallographic preferred orientation of matrix containing a dispersed second phase: insights from experimentally deformed ice. Earth Planet. Sci. Lett., 449, 272-281

Dahl-Jensen D, Thorsteinsson T, Alley RB and Shoji H (1997) Flow properties of the ice from the Greenland Ice Core Project. J. Geophys. Res., 102(C12), 26831-26840
De Bresser JHP, Peach CJ, Reijs JPJ and Spiers CJ (1998) On dynamic recrystallization during solid state flow: effects of stress and temperature. Geophys. Res. Lett., 25, 3457-3460

den Brok B (1996) The effect of crystallographic orientation on pressure solution in quartzite. J. Struct. Geol., 18(6), 859-860

Derby B and Ashby MF (1987) On dynamic recrystallization. Scr. Mater., 21, 879-884

Durand $G$ and 10 others (2006) Effect of impurities on grain growth in cold ice sheets. J. Geophys. Res. 111(F01015) doi: 10.1029/ 2005JF000320

Durham WB, Kirby SH, Heard HC, Stern LA and Boro CO (1988) Water ice phases II, III, and V: plastic deformation and phase relationships. J. Geophys. Res., 93, 10191-10208

Durham WB, Kirby SH and Stern LA (1992) Effects of dispersed particulates on the rheology of water ice at planetary conditions. J. Geophys. Res., 97, 20883-20897

Duval P and Le Gac H (1980) Does the permanent creep-rate of polycrystalline ice increase with crystal size? J. Glaciol., 25(91), 151-157

Eichler J and 7 others (2017) Location and distribution of microinclusions in the EDML and NEEM ice cores using optical microscopy and in situ Raman spectroscopy. Cryosphere, 11, 10751090

Fisher DA and Koerner RM (1986) On the special rheological properties of ancient microparticle-laden Northern Hemisphere ice as derived from bore-hole and core measurements. J. Glaciol., 32, $501-510$

Gagliardini O and 14 others (2013) Capabilities and performance of Elmer/lce, a new-generation ice sheet model. Geosci. Model Dev., 6, 1299-1318

Gillet-Chaulet F, Gagliardini O, Meyssonnier J, Zwinger T and Ruokolainen J (2006) Flow-induced anisotropy in polar ice and related ice-sheet flow modelling. J. Nonnewton. Fluid Mech., 134, 33-43

Gow AJ and Williamson T (1976) Rheological implications of the internal structure and crystal fabrics of the West Antarctic ice sheet as revealed by deep core drilling at Byrd Station. CRREL Rep., 76, 1665-1677

Gow AJ and 6 others (1997) Physical and structural properties of the Greenland Ice Sheet Project 2 ice core: a review. J. Geophys. Res., 102, 26559-26575

Hammes DM and Peternell M (2016) FAME: software for analysing rock microstructures. Comput. Geosci., 90, 23-33

Handel R, Davidchack RL, Anwar J and Brukhno J (2008) Direct calculation of solid-liquid interfacial free energy for molecular systems. TIP4P ice-water interface. Phys. Rev. Lett., 100, 036104

Herwegh M and Berger A (2004) Deformation mechanisms in second-phase affected microstructures and their energy balance. J. Struct. Geol., 26, 1483-1498

Hielscher R and Schaeben H (2008) A novel pole figure inversion method: specification of the MTEX algorithm. J. Appl. Crystallogr., 41(6), 1024-1037

Hooke RL, Dahlin BB and Kauper T (1972) Creep of ice containing dispersed fine sand. J. Glaciol., 11(63), 327-336

Hunter NJR, Wilson CJL and Luzin V (2017) Comparison of textures from fabric analyser, electron backscatter diffraction, and neutron diffraction techniques. J. Microsc., 265, 169-184.

Jacka TH and Li J (2000) Flow rates and crystal orientation fabrics in compression of polycrystalline ice at low temperature and stresses. In Hondoh T ed. Physics of ice core records I. Hokkaido University Press, Sapporo, 83-113

Jacka TH, Donoghue S, Li J, Budd WF and Anderson RM (2003) Laboratory studies of the flow rates of debris-laden ice. Ann. Glaciol., 37, 108-112

Joughin I, Smith BE, Howat IM, Scambos T and Moon T (2010) Greenland flow variability from ice-sheet-wide velocity mapping. J. Glaciol., 56, 415-430

Kamb WB (1959) Theory of preferred orientation development by crystallisation under stress. J. Geol., 67, 153-170 
Kamb WB and Engelhardt $\mathrm{H}$ (1987) Waves of accelerated motion in a glacier approaching surge. The mini-surges of Variegated Glacier, Alaska, USA. J. Glaciol., 33, 27-46

Kipfstuhl S and 8 others (2009) Evidence of dynamic recrystallization in polar firn. J. Geophys. Res., 114(B5) (doi: 10.1029/ 2008JB005583)

Kirstein O, Luzin $V$ and Garbe U (2009) The strain-scanning diffractometer Kowari. Neutron News 20, 34-36

Li J, Jacka TH and Morgan V (1998) Crystal-size and microparticle record in the ice core from Dome Summit South, Law Dome, East Antarctica. Ann. Glaciol., 35, 343-348

Mainprice D, Bachmann F, Hielscher R and Schaeben H (2014) Descriptive tools for the analysis of texture projects with large data sets using MTEX: strength, symmetry and components. Geol. Soc. Spec. Publ., 409, 251-271 (doi. org/10.1144/SP409.8)

Martin C, Gudmundsson GH, Pritchard HD and Gagliardini O (2009) On the effects of anisotropic rheology on ice flow, internal structure, and the age-depth relationship at ice divides. J. Geophys. Res., 114, F04001 (doi: 10.1029/2008JF001204)

Martin C, Gudmundsson GH and King EC (2014) Modelling of Kealey Ice Rise, Antarctica, reveals stable ice-flow conditions in East Ellsworth Land over millennia. J. Glaciol., 60(219), 139-146

Matthies S, Vinel GW and Helming K (1987) Standard distributions in texture analysis. Akademie-Verlag, Berlin, Germany

McDaniel S, Bennett K, Durham WB and Waddington ED (2006) In situ deformation apparatus for time-of-flight neutron diffraction: texture development of polycrystalline ice Ih. Rev. Sci. Instrum., 77, 093902

Middleton CA, Grindrod PM and Sammonds PR (2017) The effect of rock particles and $\mathrm{D}$ replacement on the flow behaviour of ice. Philos. Trans., A375, 20150349 (doi: 10.1098/rsta.2015.0349)

Moore PL (2014) Deformation of debris-ice mixtures. Rev. Geophys., 52, 435-467 (doi: 10.1002/2014RG000453)

Nayar HS, Lenel FV and Ansell GS (1971) Creep of dispersions of ultra-fine amorphous silica. J. Appl. Phys., 42(10), 3786-3789

Paterson WSB (1977) Secondary and tertiary creep of glacier ice as measured by borehole closure rates. Rev. Geophys. Space Phys., 15(1), 47-55

Peternell M and Wilson CIL (2016) Effect of strain rate cycling on microstructures and crystallographic preferred orientation during high-temperature creep. Geology, 44, 279-282

Peternell M and 5 others (2009) A new approach to crystallographic orientation measurement for apatite fission track analysis: effects of crystal morphology and implications for automation. Chem. Geol., 265(3-4), 527-539 (doi. org/10.1016/j.chemgeo.2009.05.021)

Peternell M, Russel-Head DS and Wilson CJL (2011) A technique for recording polycrystalline structure and orientation during in situ deformation cycles of rock analogues using an automated fabric analyser. J. Microsc., 242, 181-188

Petrenko VF and Whitworth RW (1999) Physics of ice. Oxford University Press, New York

Pettit EC, Thorsteinsson T, Jacobson HP and Waddington ED (2007) The role of crystal fabric in flow an ice divide. J. Glaciol., 53(181), 277-288

Piazolo S, Wilson CJL, Luzin V, Brouzet C and Peternell M (2013) Dynamics of ice mass deformation: linking processes to rheology, texture and microstructure. G-Cubed, 14, 4185-4194 (doi: 10.1002/ggge.20246)

Qi C, Goldsby DL and Prior DJ (2017) The down-stress transition from cluster to cone fabrics in experimentally deformed ice. Earth Planet. Sci. Lett., 471, 136-147

Sayles FH (1968) Creep of frozen sands. CRREL Technical Report 190. U.S. Army Cold Regions Research and Engineering Laboratory, Hanover, New Hampshire

Scheidegger AE (1965) On the statistics of the orientation of bedding planes, grain axes, and similar sedimentological data. US Geol. Surv. Prof. Paper, 525-C, 1-230

Schulson EM and Duval P (2009) Creep and fracture of ice. Cambridge University Press, Cambridge
Shoji $\mathrm{H}$ and Langway CC (1984) Flow behaviour of basal ice as related to modelling considerations. Ann. Glaciol., 5, 141-148

Shoji H and Langway CC (1988) Flow-law parameters of the Dye 3, Greenland, deep ice core. Ann. Glaciol., 10, 146-150

Song M, Baker I and Cole DM (2005) The effect of particles on dynamic recrystallization and fabric development of granular ice during creep. J. Glaciol., 51(174), 377-382

Song M, Baker I and Cole DM (2008) The effect of particles on creep rate and microstructures of granular ice. J. Glaciol., 54 (186), 533-537

Soule D and Nezbeda C (1968) Direct basal-plane shear in singlecrystal graphite. J. Appl. Phys., 39, 5122-5139

Sturcken EF and Croach JW (1963) Predicting physical properties in oriented metals. Trans. Metall. Soc. AIME, 227, 934-940

Tison J-L, Thorsteinsson T, Lorrain RD and Kipfstuhl J (1994) Origin and development of textures and fabrics in basal ice at Summit, Central Greenland. Earth Planet. Sci. Lett., 125, 421-437

Thorsteinsson T, Kipfstuhl J and Miller H (1997) Textures and fabrics in the GRIP ice core. J. Geophys. Res., 102(C12), 26583-26599 (doi: 10.1029/97JC00161)

Thorsteinsson T, Waddington ED, Taylor KC, Alley RB and Blankenship DD (1999) Strain-rate enhancement at Dye 3, Greenland. J. Glaciol., 45(150), 338-345

Thwaites RJ, Wilson CJL and McCray AP (1984) Relationship between bore hole closure and crystal fabrics in Antarctic ice core from Cape Folger. J. Glaciol., 30(105), 171-179

Urai JL, Means WD and Lister GS (1986) Dynamic recrystallization of minerals. In Heard HC and Hobbs BE eds. Mineral and rock deformation: laboratory studies. The Paterson volume. Am Geophys. Union, Washington DC, 161-199. (doi. org/10.1029/ GM036p0247)

Vaughan DG (1993) Relating the occurrence of crevasses to surface strain rates. J. Glaciol., 39(132), 255-266

Vollmer FW (1990) An application of eigenvalue methods to structural domain analysis. Geol. Soc. Am. Bull., 102, 786-791

Wenk HR (2006) Neutron diffraction texture analysis. Rev. Mineral. Geochem., 63, 399-426

Wilson CIL and Peternell M (2011) Evaluating ice fabrics using fabric analyser techniques in Sørsdal Glacier, East Antarctica. J. Glaciol. 57(205), 881-894

Wilson CJL and Russell-Head DS (1982) Steady-state preferred orientation of ice deformed in plane strain at $-1{ }^{\circ} \mathrm{C}$. J. Glaciol., 28(98), 145-160

Wilson CJL and Zhang Y (1994) Comparison between experimental and computer modelling of plane-strain simple-shear ice deformation. J. Glaciol., 40(134), 46-55.

Wilson CJL, Russell-Head DS and Sim HM (2003) The application of an automated fabric analyser system to the textural evolution of folded ice layers in shear zones. Ann. Glaciol., 37, 7-17

Wilson CJL, Russell-Head DS, Kunze K and Viola G (2007) The analysis of quartz c-axis fabrics using a modified optical microscope. J. Microsc., 227(1), 30-41

Wilson CJL, Peternell M, Piazolo S and Luzin V (2014) Microstructure and fabric development in ice: lessons learned from in situ experiments and implications for understanding rock evolution. J. Struct. Geol., 61, 50-77

Woodcock NH (1977) Specification of fabric shapes using an eigenvalue method. Geol. Soc. Am. Bull., 88, 1231-1236

Yasui M and Arakawa M (2008) Experimental study on the rate dependent strength of ice-silica mixture with silica volume fractions up to 0.63. Geophys. Res. Lett., 35, L12206 (doi: 10.1029/ 2008GL033787)

\section{APPENDIX \\ EVALUATION OF EXPERIMENTAL TECHNIQUES}

Complementary fabric analyser and neutron diffraction data were obtained from most samples to: (1) compare [c]-axis CPOs; and (2) understand microstructural evolution from 
optical observations and undertake grain size analysis. Comparative analyses of [c]-axis patterns (Figs 2-5) show that the best qualitative and quantitative means of establishing the CPO was by neutron diffraction (Fig. 6), as it has 3-D data acquisition and larger sample volume (Hunter and others, 2017). Whereas, the fabric analyser technique was suited for grain-scale analyses, where individual orientations could be linked to their source grains. However, the fabric analyser's efficiency for gathering orientation data from highly undulose grains is limited by geometrical and retardation quality values (Peternell and others, 2009) and some caution needed to be undertaken. This was particularly the case with ice-graphite samples, where graphite can underlie the ice crystals inhibiting geometrical and retardation quality. In contrast, the fabric analyser was an excellent tool in capturing thin-section images made from the deformed sample.

In undertaking the 2-D experiments, using optical techniques (Wilson and others, 2007), the field of view $(5 \times 5 \mathrm{~mm})$ was limited to only a portion of the sample which is $1.5 \mathrm{~cm} \times$ $3 \mathrm{~cm}$ and 250-300 $\mu \mathrm{m}$ thick. The optical data had a resolution of $5 \mu \mathrm{m} /$ pixel and produced excellent grain maps so that microstructural features could be coupled to orientations. As these were plane strain experiments, with the sample constrained by two glass plates, some grains will have a high Schmid factor (Azuma, 1995), with a hardening component that depends on the angle between the grains $[c]$-axis orientation and the glass plate. For this reason, many grains undergo inhomogeneous stress distributions on the inter-grain and intra-grain scales (Wilson and Zhang, 1994) and this may partially explain the bimodal grain sizes and more random CPOs (Figs 8c, d). As pointed out by Wilson and Zhang (1994), the triple-point junction in a grain with an easy-glide orientation is often migrating at the expense of grains in hard-glide orientations and this may explain the weaker CPOs.

A problem pointed out by Woodcock (1977) is that when representing changes in $\mathrm{CPO}$ evolution for 2-D data using the eigenvector method on PGR diagrams (Vollmer, 1990), we cannot adequately represent certain types of multimodal data. For this reason, the evolution of small-circle cones (Figs 13a-d) seen in an equal-area stereonet plots would wrongly return a random result from similar data sets if plotted on a PGR diagram (Figs 14e-g). For this reason, we recommend inspecting the form of the data on an equalarea plot before accepting the PGR results. 